\title{
An optimized SPDMFE extrapolation approach based on the POD technique for 2D viscoelastic wave equation
}

\section{Zhendong Luo ${ }^{1 *}$ and Fei Teng ${ }^{2}$}

"Correspondence: zhdluo@163.com 'School of Mathematics and Physics, North China Electric Power University, No. 2, Bei Nong Road, Changping District, Beijing, 102206, China

Full list of author information is available at the end of the article

\begin{abstract}
An optimized splitting positive definite mixed finite element (SPDMFE) extrapolation approach based on proper orthogonal decomposition (POD) technique is developed for the two-dimension viscoelastic wave equation (2DVWE). The errors of the optimized SPDMFE extrapolation solutions are analyzed. The implement procedure for the optimized SPDMFE extrapolation approach is offered. Some numerical simulations have verified that the numerical conclusions are accordant with theoretical ones. This implies that the optimized SPDMFE extrapolation approach is viable and valid for solving 2DVWE.
\end{abstract}

MSC: 74S10;65M15;35Q35

Keywords: optimized splitting positive definite mixed finite element extrapolation approach; proper orthogonal decomposition technique; viscoelastic wave equation; error estimate; numerical simulation

\section{Introduction}

In this article, we study the following two-dimensional viscoelastic wave equation (2DVWE).

Problem I For $0<t<T$, find $u$ that satisfies

$$
\left\{\begin{array}{l}
u_{t t}-\varepsilon \Delta u_{t}-\gamma \Delta u=f, \quad \text { in } \Omega, \\
u(x, y, t)=\psi(x, y, t), \quad \text { on } \partial \Omega, \\
u(x, y, 0)=\psi_{0}(x, y), \quad u_{t}(x, y, 0)=\psi_{1}(x, y), \quad \text { in } \Omega,
\end{array}\right.
$$

where $\Omega \subset \mathbf{R}^{2}$ is a bounded convex polygonal domain with the boundary $\partial \Omega, u_{t t}=\partial^{2} u / \partial t^{2}$, $u_{t}=\partial u / \partial t, \varepsilon$ and $\gamma$ are two positive coefficients, $f(x, y, t), \psi(x, y, t), \psi_{0}(x, y)$, and $\psi_{1}(x, y)$ are all given functions, and $T$ is the final time. For convenience and without losing universality, we assume that $\psi(x, y, t)=\psi_{0}(x, y)=\psi_{1}(x, y)=0$ and $\varepsilon=\gamma=1$ in the following discussion.

The main motivation and physical background of 2DVWE (1) are the modeling of the wave propagation and vibration phenomena in the viscoelastic matter (see, e.g., [1, 2]). Although there have been several numerical methods for 2DVWE (see, e.g., [3-5]), the splitting positive definite mixed finite element (SPDMFE) approach in [6] is one of most novel

(C) The Author(s) 2017. This article is distributed under the terms of the Creative Commons Attribution 4.0 International License (http://creativecommons.org/licenses/by/4.0/), which permits unrestricted use, distribution, and reproduction in any medium, provided you give appropriate credit to the original author(s) and the source, provide a link to the Creative Commons license, and indicate if changes were made. 
ones for dealing with 2DVWE because it cannot only keep away from the restriction of the Brezzi-Babuška inequality and simultaneously find an unknown function (displacement) and its gradient (stress), but it can also ensure that the full discrete SPDMFE formulation is positive definite and robust. Reference [7] has established a new SPDMFE formulation that includes fewer degrees of freedom than those and is different from that in [6], but it still includes lots of degrees of freedom. Hence, a major key issue is how to lessen the degrees of freedom for the new SPDMFE formulation in [7] so as to reduce the calculating load and the operation time in the numerical computation as well as obtain a desired accurate SPDMFE solution.

Many reports have proven that the proper orthogonal decomposition (POD) technique is one of the most valid approaches lessening the degrees of freedom (i.e., unknowns) of numerical models for the time-dependent PDEs and alleviating the truncated error accumulation in the calculating course (see [8-10]). In fact, the POD technique offers an orthogonal basis to the given data, i.e., offers an optimal low order approximation to the given data.

Though some optimized numerical formulations based on the POD technique for the time-dependent PDEs were presented (see [11-16]), these optimized formulations utilize all classical numerical solutions on the whole time interval $[0, T]$ to formulate the POD bases and the optimized models, before recomputing the solutions on the same time interval $[0, T]$, which actually belongs to the repeated calculations on the same time interval $[0, T]$.

In order to eliminate those unrewarding repeated computations in the reduced-order finite element (FE) methods based on the POD technique, several reduced-order extrapolation FE methods based on the POD technique for hyperbolic equations, Sobolev equations, and the non-stationary parabolized Navier-Stokes equations have successfully been proposed by Luo et al. since 2014 (see [17-19]). Nevertheless, as far as we know, there is not any article treating that the optimized SPDMFE extrapolation approach based on the POD technique for 2DVWE is set up or the implement procedure for the optimized SPDMFE extrapolation approach is offered. Therefore, in this article, we set up the optimized SPDMFE extrapolation approach based on the POD technique for 2DVWE and offer the error estimates for the optimized SPDMFE extrapolation solutions and the implement procedure for the optimized SPDMFE extrapolation approach. We adopt some numerical simulations to verify that the optimized SPDMFE extrapolation approach is viable and valid for dealing 2DVWE, too.

The rest of the article is as follows. Section 2 sets up the classical SPDMFE formulation for 2DVWE and extracts the snapshots. In Section 3, we construct the POD bases and build the optimized SPDMFE extrapolation approach containing very few unknowns but having the desired accuracy for 2DVWE. In Section 4, we offer the error estimates for the optimized SPDMFE extrapolation solutions and the implement procedure for the optimized SPDMFE extrapolation approach. In Section 5, we adopt some numerical simulations to verify that the numerical conclusions are accordant with theoretical ones, validating the feasibility and efficiency of the optimized SPDMFE extrapolation approach for finding the numerical solutions of 2DVWE. Section 6 offers main conclusions.

\section{Classical SPDMFE formulation and formulation of snapshots}

The Sobolev spaces used in the following belong to standard (see [20]). The natural inner product in $\left[L^{2}(\Omega)\right]^{d}(d=1,2,4)$ is denoted by $(\cdot, \cdot)$ and the norms all are represented by 
$\|\cdot\|_{0}$. The divergence space used in this context is defined by

$$
\boldsymbol{W}=H(\operatorname{div} ; \Omega)=\left\{\boldsymbol{q} \in L^{2}(\Omega)^{2} ; \operatorname{div} \boldsymbol{q} \in L^{2}(\Omega)\right\}
$$

with norm $\|\boldsymbol{q}\|_{\boldsymbol{w}}=\left[\|\boldsymbol{q}\|_{0}^{2}+\|\operatorname{div} \boldsymbol{q}\|_{0}^{2}\right]^{1 / 2}$. Let $U=L^{2}(\Omega)$. Put $\boldsymbol{p}=\nabla u$. Then it follows from Problem I that $u_{t t}=-\operatorname{div}\left(\boldsymbol{p}_{t}+\boldsymbol{p}\right)+f$ and $\boldsymbol{p}_{t t}=-\nabla\left(u_{t t}\right)=\nabla \operatorname{div}\left(\boldsymbol{p}_{t}\right)+\nabla \operatorname{div}(\boldsymbol{p})-\nabla f$. Thus, the splitting positive definite mixed weak formulation for Problem I may be expressed by the following.

Problem II Find $(u, \boldsymbol{p}) \in U \times \boldsymbol{W}$ that satisfies, for any $t \in(0, T)$,

$$
\left\{\begin{array}{l}
\left(u_{t t}, v\right)+\left(\operatorname{div} \boldsymbol{p}_{t}, v\right)+(\operatorname{div} \boldsymbol{p}, v)=(f, v), \quad \forall v \in U, \\
\left(\boldsymbol{p}_{t t}, q\right)+\left(\operatorname{div} \boldsymbol{p}_{t}, \operatorname{div} \boldsymbol{q}\right)+(\operatorname{div} \boldsymbol{p}, \operatorname{div} \boldsymbol{q})=(f, \operatorname{div} \boldsymbol{q}), \quad \forall \boldsymbol{q} \in \boldsymbol{W}, \\
u(x, y, 0)=u_{t}(x, y, 0)=0, \quad \boldsymbol{p}(x, y, 0)=\boldsymbol{p}_{t}(x, y, 0)=\mathbf{0}, \quad(x, y) \in \Omega .
\end{array}\right.
$$

The following result for Problem II has been proved in [7] or can be proved by using the same technique as that in [6].

Theorem 1 Iff $\in L^{2}\left(0, T ; L^{2}(\Omega)\right)$, then for Problem II there exists a unique solution $(u, \boldsymbol{p}) \in$ $U \times \boldsymbol{W}$ that satisfies

$$
\begin{aligned}
& \left\|\boldsymbol{p}_{t}\right\|_{0}^{2}+\left\|\operatorname{div} \boldsymbol{p}_{t}\right\|_{L^{2}\left(L^{2}\right)}^{2}+\|\operatorname{div} \boldsymbol{p}\|_{0}^{2} \leq\|f\|_{L^{2}\left(L^{2}\right)}^{2}, \\
& \left\|u_{t}\right\|_{0}^{2} \leq(2+T)\|f\|_{L^{2}\left(L^{2}\right)}^{2} \exp (T)
\end{aligned}
$$

where $\|\cdot\|_{W^{m, r_{1}}\left(W^{\left.l, r_{2}\right)}\right.}$ is the norm in $W^{m, r_{1}}\left(0, T ; W^{m, r_{2}}(\Omega)\right)$ or $W^{m, r_{1}}\left(0, T ; W^{m, r_{2}}(\Omega)^{2}\right)^{2}(1 \leq$ $\left.r_{1}, r_{2} \leq \infty\right)$.

Let $N$ represent a positive integer, $k=T / N$ the time step increment, and $g^{n}$ the semidiscrete approximation for $g\left(x, y, t_{n}\right)$ with respect to time at $t=t_{n}$. Write

$$
g^{n, \frac{1}{2}}=\frac{g^{n+1}+g^{n-1}}{2}, \quad \bar{\partial}_{t} g^{n}=\frac{g^{n+1}-g^{n}}{k}, \quad \bar{\partial}_{t} \bar{\partial}_{t} g^{n}=\frac{g^{n+1}-2 g^{n}+g^{n-1}}{k^{2}} .
$$

Thus, the splitting positive definite semi-discrete model about time $t$ for Problem I may be expressed in the following.

Problem III Find $\left(u^{n+1}, \boldsymbol{p}^{n+1}\right) \in U \times \boldsymbol{W}(1 \leq n \leq N-1)$ such that

$$
\left\{\begin{array}{l}
\left(\bar{\partial}_{t} \bar{\partial}_{t} u^{n}, v\right)+\left(\operatorname{div}\left(\bar{\partial}_{t} \boldsymbol{p}^{n}+\bar{\partial}_{t} \boldsymbol{p}^{n-1}\right), v\right)+\left(\operatorname{div} \boldsymbol{p}^{n, \frac{1}{2}}, v\right)=\left(f\left(t_{n}\right), v\right), \quad \forall v \in U, \\
\left(\bar{\partial}_{t} \bar{\partial}_{t} \boldsymbol{p}^{n}, \boldsymbol{q}\right)+\left(\operatorname{div}\left(\bar{\partial}_{t} \boldsymbol{p}^{n}+\bar{\partial}_{t} \boldsymbol{p}^{n-1}\right), \operatorname{div} \boldsymbol{q}\right)+\left(\operatorname{div} \boldsymbol{p}^{n, \frac{1}{2}}, \operatorname{div} \boldsymbol{q}\right) \\
=\left(f\left(t_{n}\right), \operatorname{div} \boldsymbol{q}\right), \quad \forall \boldsymbol{q} \in \boldsymbol{W}, \\
u^{0}=u^{1}=0, \quad \boldsymbol{p}^{0}=\boldsymbol{p}^{1}=\mathbf{0}, \quad(x, y) \in \Omega .
\end{array}\right.
$$

Existence, uniqueness, stability, and convergence (error estimates) of solutions of Problem III have been provided in [7] or can be proved by using the same technique as that in [6]. 
Theorem 2 Under the assumptions of Theorem 1, Problem III there exist a unique set of solutions $\left(u^{n}, \boldsymbol{p}^{n}\right) \in U \times \boldsymbol{W}(1 \leq n \leq N)$ that satisfy

$$
\left\|u^{n}\right\|_{0}+\left\|\boldsymbol{p}^{n}\right\|_{0}+\left\|\operatorname{div} \boldsymbol{p}^{n}\right\|_{0} \leq \tilde{C}\|f\|_{L^{\infty}\left(L^{2}\right)}
$$

where $\tilde{C}=2\left(T^{2}+2 \sqrt{T^{5}}+\sqrt{T^{3}}+\sqrt{T}\right)$ is a constant. And if the solutions $(u, \boldsymbol{p}) \in$ $H^{4}(0, T ; U) \times\left[H^{4}\left(0, T ; U^{2}\right)^{2} \cap H^{3}\left(0, T ; H^{1}(\Omega)^{2}\right)^{2}\right]$ for Problem II, then we have

$$
\left\|\boldsymbol{p}\left(t_{n}\right)-\boldsymbol{p}^{n}\right\|_{0}+\left\|\operatorname{div}\left(\boldsymbol{p}\left(t_{n}\right)-\boldsymbol{p}^{n}\right)\right\|_{0}+\left\|u\left(t_{n}\right)-u^{n}\right\|_{0} \leq C k^{2}, \quad 1 \leq n \leq N,
$$

where $C$ used in the following represents a generic positive real number that is only reliant on $\|u\|_{H^{4}\left(L^{2}\right)},\|\boldsymbol{p}\|_{H^{4}\left(L^{2}\right)}$, and $\|\boldsymbol{p}\|_{H^{3}\left(H^{1}\right)}$, namely $\|f\|_{H^{2}\left(L^{2}\right)}$, but is not reliant on the time step $k$ and the next spatial mesh parameters $h$ and may be different at their occurrences.

Let $\Im_{h}=\{K\}$ denote a quasi consistent triangulation of $\Omega$ with $h=\max h_{K}$, here $h_{K}$ indicates the diameter of the element $K \in \Im_{h}$ (see [21] or [22]). Take the FE spaces of $U$ and $\boldsymbol{W}$ as follows:

$$
U_{h}=\left\{v_{h} \in U ;\left.v_{h}\right|_{K} \in P_{m}(K), \forall K \in \Im_{h}\right\}, \quad \boldsymbol{W}_{h}=\left\{\boldsymbol{\tau}_{h} \in \boldsymbol{W} ;\left.\boldsymbol{\tau}_{h}\right|_{K} \in \boldsymbol{P}_{K}, \forall K \in \Im_{\Im_{h}}\right\},
$$

where $m$ is a positive integer, $P_{m}(K)$ the $m$ th polynomials space on $K$, and $\boldsymbol{P}_{K}$ the R-T space of degree $\leq m$ on $K$ (see $[7,21,22])$. Then the SPDMFE formulation for Problem I may be stated as follows.

Problem IV Find $\left(u_{h}^{n+1}, \boldsymbol{p}_{h}^{n+1}\right) \in U_{h} \times \boldsymbol{W}_{h}(1 \leq n \leq N-1)$ that satisfy

$$
\left\{\begin{array}{l}
\left(\bar{\partial}_{t} \bar{\partial}_{t} u_{h}^{n}, v_{h}\right)+\left(\operatorname{div}\left(\bar{\partial}_{t} \boldsymbol{p}_{h}^{n}+\bar{\partial}_{t} \boldsymbol{p}_{h}^{n-1}\right), v_{h}\right)+\left(\operatorname{div} \boldsymbol{p}_{h}^{n, \frac{1}{2}}, v_{h}\right)=\left(f\left(t_{h}\right), v_{h}\right), \quad \forall v_{h} \in U_{h}, \\
\left(\bar{\partial}_{t} \bar{\partial}_{t} \boldsymbol{p}_{h}^{n}, \boldsymbol{q}_{h}\right)+\left(\operatorname{div}\left(\bar{\partial}_{t} \boldsymbol{p}_{h}^{n}+\bar{\partial}_{t} \boldsymbol{p}_{h}^{n-1}\right), \operatorname{div} \boldsymbol{q}_{h}\right)+\left(\operatorname{div} \boldsymbol{p}_{h}^{n, \frac{1}{2}}, \operatorname{div} \boldsymbol{q}_{h}\right) \\
\quad=\left(f\left(t_{h}\right), \operatorname{div} \boldsymbol{q}_{h}\right), \quad \forall \boldsymbol{q}_{h} \in \boldsymbol{W}_{h}, \\
u_{h}^{0}=u_{h}^{1}=0, \quad \boldsymbol{p}_{h}^{0}=\boldsymbol{p}_{h}^{1}=\mathbf{0}, \quad(x, y) \in \Omega
\end{array}\right.
$$

Existence, uniqueness, stability, and convergence (error estimate) of solutions to Problem IV have been provided in [7] or can be proved by using the same technique as that in [6].

Theorem 3 Under the assumptions of Theorems 1 and 2, Problem IV has only a set of solutions $\left\{\left(u_{h}^{n}, \boldsymbol{p}_{h}^{n}\right): 1 \leq n \leq N\right\} \subset U_{h} \times \boldsymbol{W}_{h}$ that satisfy

$$
\left\|u_{h}^{n}\right\|_{0}+\left\|\boldsymbol{p}_{h}^{n}\right\|_{0}+\left\|\operatorname{div} \boldsymbol{p}_{h}^{n}\right\|_{0} \leq \tilde{C}\|f\|_{L^{\infty}\left(L^{2}\right)}, \quad 1 \leq n \leq N,
$$

where $\tilde{C}$ is the same as that in (5), which shows that the solutions of Problem IV are stable and continuously reliant on the given functions $f(x, y, t), \psi_{0}(x, y)$, and $\psi_{1}(x, y)$ when they are nonzero. Moreover, when the solution $(u, \boldsymbol{p}) \in W^{4, \infty}\left(0, T ; H^{m+1}(\Omega)\right) \times$ $W^{4, \infty}\left(0, T ; H^{m+1}(\Omega)^{2}\right)^{2}$ for Problem II, the errors between the solution $u(t)$ to Problem I 
and the solutions $u_{h}^{n}$ to Problem IV satisfy the following estimates:

$$
\begin{aligned}
& \left\|\boldsymbol{p}\left(t_{n}\right)-\boldsymbol{p}_{h}^{n}\right\|_{0}+\left\|\operatorname{div}\left(\boldsymbol{p}\left(t_{n}\right)-\boldsymbol{p}_{h}^{n}\right)\right\|_{0}+\left\|u\left(t_{n}\right)-u_{h}^{n}\right\|_{0} \leq C(f)\left(k^{2}+h^{m+1}\right), \\
& 1 \leq n \leq N
\end{aligned}
$$

where $C(f)$ is a constant that is only reliant on $f$ and $T$ but not reliant on $h$ and $k$.

Remark 1 If only the coefficients $\varepsilon$ and $\gamma$, the functions $f(x, y, t), \psi(x, y, t), \psi_{0}(x, y)$, and $\psi_{1}(x, y)$, and $k$ and $h$ are designated, we could obtain an ensemble of solutions $\left\{\left(u_{h}^{n}, \boldsymbol{p}_{h}^{n}\right)\right.$ : $1 \leq n \leq N\}$ from Problem IV.

\section{Formulations of the POD bases and the optimized SPDMFE extrapolation approach}

We extract the first $L$ solutions $\left(u_{h}^{i}(x, y), \boldsymbol{p}_{h}^{i}(x, y)\right)(1 \leq i \leq L)$ (usually, $L \ll N$, say, $L=20$, $N=200)$ from the solution set $\left\{\left(u_{h}^{n}, \boldsymbol{p}_{h}^{n}\right): 1 \leq n \leq N\right\}$ of Problem IV in Section 2 as the snapshots. Let $\boldsymbol{V}_{i}=\left(u_{h}^{i}, \boldsymbol{p}_{h}^{i}\right)^{T}(1 \leq i \leq L)$,

$$
\mathcal{V}=\operatorname{span}\left\{\boldsymbol{V}_{1}, \boldsymbol{V}_{2}, \ldots, \boldsymbol{V}_{L}\right\}
$$

and $\left\{\boldsymbol{\varphi}_{j}\right\}_{j=1}^{l}$ represent a set of standard orthogonal basis of $\mathcal{V}$ with $l=\operatorname{dim} \mathcal{V}(l \leq L)$. Thus, the elements $\boldsymbol{V}_{i}$ can indicate as follows:

$$
\boldsymbol{V}_{i}=\sum_{j=1}^{l}\left(\boldsymbol{V}_{i}, \boldsymbol{\varphi}_{j}\right)_{\tilde{u}} \boldsymbol{\varphi}_{j}, \quad 1 \leq i \leq L .
$$

The above $\tilde{\boldsymbol{U}}=U \times \boldsymbol{W}$ and $\left(\boldsymbol{V}_{i}, \boldsymbol{\varphi}_{j}\right)_{\tilde{\boldsymbol{u}}}=\left(u_{h}^{i}, \varphi_{u j}\right)+\left(\boldsymbol{p}_{h}^{i}, \boldsymbol{\varphi}_{p j}\right)+\left(\operatorname{div} \boldsymbol{p}_{h}^{i}\right.$, $\left.\operatorname{div} \boldsymbol{\varphi}_{p j}\right)$, while $\varphi_{u j}$ and $\boldsymbol{\varphi}_{p j}$ are the orthonormal bases associated with $u$ and $\boldsymbol{p}$, separately.

Definition 1 The POD approach is just to find a set of standard orthogonal basis $\left\{\varphi_{i}: 1 \leq\right.$ $i \leq l\}$ that meet

$$
\min _{\left\{\boldsymbol{\varphi}_{j}\right\}_{j=1}^{d}} \frac{1}{L} \sum_{i=1}^{L}\left\|\boldsymbol{V}_{i}-\sum_{j=1}^{d}\left(\boldsymbol{V}_{i}, \boldsymbol{\varphi}_{j}\right)_{\tilde{\boldsymbol{U}}} \boldsymbol{\varphi}_{j}\right\|_{\tilde{\boldsymbol{U}}}^{2}
$$

and

$$
\left(\varphi_{u i}, \varphi_{u j}\right)=\delta_{i j}, \quad\left(\varphi_{p i}, \varphi_{p j}\right)_{\boldsymbol{w}}=\delta_{i j}, \quad i=1,2, \ldots, d, j=1,2, \ldots, i
$$

where $\left\|\boldsymbol{V}_{i}\right\|_{\tilde{\boldsymbol{u}}}^{2}=\left\|u_{h}^{i}\right\|_{0}^{2}+\left\|\boldsymbol{p}_{h}^{i}\right\|_{0}^{2}+\left\|\operatorname{div} \boldsymbol{p}_{h}^{i}\right\|_{0}^{2}$. A set of solutions $\left\{\boldsymbol{\varphi}_{j}: 1 \leq j \leq d\right\}$ for the formulas (13) and (14) are referred as a set of POD bases with rank $d$.

Now, we make up a correlation matrix $\boldsymbol{A}=\left(A_{i j}\right)_{L \times L} \in R^{L \times L}$ associated with the snapshots $\left\{\boldsymbol{V}_{i}\right\}_{i=1}^{L}$ via $A_{i j}=\left(\boldsymbol{V}_{i}, \boldsymbol{V}_{j}\right) \tilde{\boldsymbol{u}} / L$. Because $\boldsymbol{A}$ is a semi-definite positive matrix having the rank $l$, the solution of (13) and (14) can be sought. Further, we have the following results (see, e.g., [14] or [15]). 
Proposition 1 Let $\lambda_{1} \geq \lambda_{2} \geq \cdots \geq \lambda_{l}>0$ indicate the positive eigenvalues for the matrix $\boldsymbol{A}$ and $\boldsymbol{v}_{1}, \boldsymbol{v}_{2}, \ldots, \boldsymbol{v}_{l}$ be the corresponding standard orthogonal eigenvectors. Thus, a POD basis with rank $d \leq l$ is obtained by

$$
\boldsymbol{\varphi}_{i}=\frac{1}{\sqrt{\lambda_{i}}} \sum_{j=1}^{L}\left(\boldsymbol{v}_{i}\right)_{j} \boldsymbol{v}_{j}=\frac{1}{\sqrt{\lambda_{i}}}\left(\boldsymbol{V}_{1}, \boldsymbol{V}_{2}, \ldots, \boldsymbol{V}_{L}\right) \boldsymbol{v}_{i}, \quad 1 \leq i \leq d
$$

where $\left(\boldsymbol{v}_{i}\right)_{j}(1 \leq j \leq L)$ indicates the $j$ th component of the standard orthogonal eigenvectors $\boldsymbol{v}_{i}$. In addition, we obtain the error formula

$$
\frac{1}{L} \sum_{i=1}^{L}\left\|\boldsymbol{V}_{i}-\sum_{j=1}^{d}\left(\boldsymbol{V}_{i}, \boldsymbol{\varphi}_{j}\right)_{\tilde{U}} \boldsymbol{\varphi}_{j}\right\|_{\tilde{\boldsymbol{U}}}^{2}=\sum_{j=d+1}^{l} \lambda_{j}
$$

Let $U^{d}=\operatorname{span}\left\{\varphi_{u 1}, \varphi_{u 2}, \ldots, \varphi_{u d}\right\}$ and $\boldsymbol{W}^{d}=\operatorname{span}\left\{\boldsymbol{\varphi}_{p 1}, \boldsymbol{\varphi}_{p 2}, \ldots, \boldsymbol{\varphi}_{p d}\right\}$. For any $u_{h} \in U_{h}$, define the $L^{2}$-operator $P^{d}: U_{h} \rightarrow U^{d}$ as follows:

$$
\left(P^{d} u_{h}, v_{d}\right)=\left(u_{h}, v_{d}\right), \quad \forall v_{d} \in V^{d} .
$$

Then, because $P^{d}$ is bounded, there exists an extension $P^{h}: U \rightarrow U_{h}$ of $P^{d}$ that meets $\left.P^{h}\right|_{U_{h}}=P^{d}: U_{h} \rightarrow U^{d}$ and, for each $u \in V, P^{h}$ meets (see [23])

$$
\left(P^{h} u-u, v_{h}\right)=0, \quad \forall v_{h} \in U_{h} .
$$

For any $\boldsymbol{p}_{h} \in \boldsymbol{W}_{h}$, define div-operator $\rho^{d}: \boldsymbol{W}_{h} \rightarrow \boldsymbol{W}^{d}$ denoted by

$$
\left(\rho^{d} \boldsymbol{p}_{h}, \boldsymbol{q}_{d}\right)+\left(\operatorname{div} \rho^{d} \boldsymbol{p}_{h}, \operatorname{div} \boldsymbol{q}_{d}\right)=\left(\boldsymbol{p}_{h}, \boldsymbol{q}_{d}\right)+\left(\operatorname{div} \boldsymbol{p}_{h}, \operatorname{div} \boldsymbol{q}_{d}\right), \quad \forall \boldsymbol{q}_{d} \in \boldsymbol{W}^{d} .
$$

Similarly, because $\rho^{d}$ is bounded, there exists an extension $\rho^{h}: \boldsymbol{W} \rightarrow \boldsymbol{W}_{h}$ of $\rho^{d}$ that meets $\rho^{h} \mid \boldsymbol{W}_{h}=\rho^{d}: \boldsymbol{W}_{h} \rightarrow \boldsymbol{W}^{d}$ and, for each $\boldsymbol{p} \in \boldsymbol{W}, \rho^{h}$ meets (see [23])

$$
\left(\rho^{h} \boldsymbol{p}, \boldsymbol{q}_{h}\right)+\left(\operatorname{div} \rho^{h} \boldsymbol{p}, \operatorname{div} \boldsymbol{p}_{h}\right)=\left(\boldsymbol{p}, \boldsymbol{q}_{h}\right)+\left(\operatorname{div} \boldsymbol{p}, \operatorname{div} \boldsymbol{q}_{h}\right), \quad \forall \boldsymbol{q}_{h} \in \boldsymbol{W}_{h} .
$$

Thanks to (18) and (20), the operators $P^{h}$ and $\rho^{h}$ are well defined and bounded (see [21] or [22])

$$
\begin{aligned}
& \left\|P^{h} u\right\|_{0} \leq\|u\|_{0}, \quad \forall u \in U, \\
& \left\|\rho^{h} \boldsymbol{p}\right\|_{0}+\left\|\operatorname{div}\left(\rho^{h} \boldsymbol{p}\right)\right\|_{0} \leq C\|\boldsymbol{p}\|_{\boldsymbol{w}}, \quad \forall \boldsymbol{p} \in \boldsymbol{W} .
\end{aligned}
$$

Further, we have the following lemma.

Lemma 1 The above operators $P^{d}$ and $\rho^{d}(1 \leq d \leq l)$ meet (see $\left.[14,15]\right)$

$$
\frac{1}{L} \sum_{i=1}^{L}\left\|u_{h}^{i}-P^{d} u_{h}^{i}\right\|_{0}^{2} \leq C h \sum_{j=d+1}^{l} \lambda_{j}
$$




$$
\frac{1}{L} \sum_{i=1}^{L}\left[\left\|\boldsymbol{p}_{h}^{i}-\rho^{d} \boldsymbol{p}_{h}^{i}\right\|_{0}^{2}+\left\|\operatorname{div}\left(\boldsymbol{p}_{h}^{i}-\rho^{d} \boldsymbol{p}_{h}^{i}\right)\right\|_{0}^{2}\right] \leq C h \sum_{j=d+1}^{l} \lambda_{j},
$$

where $\left(u_{h}^{i}, \boldsymbol{p}_{h}^{i}\right) \in \mathcal{V}(1 \leq i \leq L)$ are the solutions to Problem V. Moreover, we have (see [21, 22])

$$
\begin{aligned}
& \left\|u-P^{h} u\right\|_{0} \leq C h^{m+1}\|u\|_{m+1}, \quad \forall u \in H^{m+1}(\Omega), \\
& \left\|\boldsymbol{p}-\rho^{h} \boldsymbol{p}\right\|_{0}+\left\|\operatorname{div}\left(\boldsymbol{p}-\rho^{h} \boldsymbol{p}\right)\right\|_{0} \leq C h^{m+1}\|\boldsymbol{p}\|_{m+1}, \quad \forall \boldsymbol{p} \in H^{m+1}(\Omega)^{2} .
\end{aligned}
$$

By means of $U^{d}$ and $\boldsymbol{W}^{d}$, the optimized SPDMFE extrapolation approach based on the POD technique is set up as follows.

Problem V Find $\left(u_{d}^{n}, \boldsymbol{p}_{d}^{n}\right) \in U^{d} \times \boldsymbol{W}^{d}$ that meets

$$
\begin{aligned}
& \left\{\begin{array}{l}
\boldsymbol{p}_{d}^{n}=\rho^{d} \boldsymbol{p}_{d}^{n}=\sum_{j=1}^{d}\left[\left(\boldsymbol{p}_{d}^{n}, \boldsymbol{\varphi}_{p j}\right) \boldsymbol{\varphi}_{p j}+\left(\operatorname{div} \boldsymbol{p}_{d}^{n}, \operatorname{div} \boldsymbol{\varphi}_{p j}\right) \boldsymbol{\varphi}_{p j}\right], \quad 1 \leq n \leq L ; \\
\left(\bar{\partial}_{t} \bar{\partial}_{t} \boldsymbol{p}_{d}^{n}, \boldsymbol{q}_{d}\right)+\left(\operatorname{div}\left(\bar{\partial}_{t} \boldsymbol{p}_{d}^{n}+\bar{\partial}_{t} \boldsymbol{p}_{d}^{n-1}\right), \operatorname{div} \boldsymbol{q}_{d}\right)+\left(\operatorname{div} \boldsymbol{p}_{d}^{n, \frac{1}{2}}, \operatorname{div} \boldsymbol{q}_{d}\right) \\
\quad=\left(f\left(t_{n}\right), \operatorname{div} \boldsymbol{q}_{d}\right), \quad \forall \boldsymbol{q}_{d} \in \boldsymbol{W}^{d}, L \leq n \leq N-1 ;
\end{array}\right. \\
& \left\{\begin{array}{c}
u_{d}^{n}=P^{d} u_{h}^{n}=\sum_{j=1}^{d}\left(u_{h}^{n}, \varphi_{u j}\right) \varphi_{u j}, \quad 1 \leq n \leq L ; \\
\left(\bar{\partial}_{t} \bar{\partial}_{t} u_{d}^{n}, v_{d}\right)+\left(\operatorname{div}\left(\bar{\partial}_{t} \boldsymbol{p}_{d}^{n}+\bar{\partial}_{t} \boldsymbol{p}_{d}^{n-1}\right), v_{d}\right)+\left(\operatorname{div} \boldsymbol{p}_{d}^{n, \frac{1}{2}}, v_{d}\right)=\left(f\left(t_{n}\right), v_{d}\right), \\
\forall v_{d} \in U^{d}, L \leq n \leq N-1 .
\end{array}\right.
\end{aligned}
$$

Let $u_{d}^{n}=\alpha_{1}^{n} \varphi_{u 1}+\alpha_{2}^{n} \varphi_{u 2}+\cdots+\alpha_{d}^{n} \varphi_{u d}$ and $\boldsymbol{p}_{d}^{n}=\beta_{1}^{n} \boldsymbol{\varphi}_{p 1}+\beta_{2}^{n} \boldsymbol{\varphi}_{p 2}+\cdots+\beta_{d}^{n} \boldsymbol{\varphi}_{p d}$. Thus, by means of Green's formula, Problem V may be restated as follows:

Problem VI Find $\left(\alpha_{1}^{n}, \alpha_{2}^{n}, \ldots, \alpha_{d}^{n}, \beta_{1}^{n}, \beta_{2}^{n}, \ldots, \beta_{d}^{n}\right)^{T} \in \mathbf{R}^{2 d}(1 \leq n \leq N)$ that meet

$$
\begin{aligned}
& \left\{\begin{array}{l}
\beta_{j}^{n}=\left(\boldsymbol{p}_{h}^{n}, \boldsymbol{\varphi}_{p j}\right)+\left(\operatorname{div} \boldsymbol{p}_{h}^{n}, \operatorname{div} \boldsymbol{\varphi}_{p j}\right), \quad 1 \leq j \leq d, 1 \leq n \leq L ; \\
\sum_{i=1}^{d} \beta_{i}^{n+1} a_{i j}=\sum_{i=1}^{d} \beta_{i}^{n}\left(\boldsymbol{\varphi}_{p i}, \boldsymbol{\varphi}_{p j}\right)-\sum_{i=1}^{d} \beta_{i}^{n-1} b_{i j}+\left(f\left(t_{n}\right), \operatorname{div} \boldsymbol{\varphi}_{p j}\right), \\
\quad 1 \leq j \leq d, L \leq n \leq N-1 ;
\end{array}\right. \\
& \left\{\begin{array}{c}
\alpha_{j}^{n}=\left(u_{h}^{n}, \varphi_{u j}\right), \quad 1 \leq j \leq d, 1 \leq n \leq L ; \\
\alpha_{j}^{n+1}=2 \alpha_{j}^{n}-\alpha_{j}^{n-1}+0.5 \sum_{i=1}^{d} k\left[(1-k) \beta_{i}^{n-1}-(k+1) \beta_{i}^{n+1}\right]\left(\operatorname{div} \boldsymbol{\varphi}_{p i}, \varphi_{u j}\right) \\
\quad+\left(f\left(t_{n}\right), \varphi_{u j}\right), \quad 1 \leq j \leq d, L \leq n \leq N-1,
\end{array}\right.
\end{aligned}
$$

where $a_{i j}=\left(\boldsymbol{\varphi}_{p i}, \boldsymbol{\varphi}_{p j}\right)+k(k+1)\left(\operatorname{div} \boldsymbol{\varphi}_{p i}, \operatorname{div} \boldsymbol{\varphi}_{p j}\right) / 2$ and $b_{i j}=\left(\boldsymbol{\varphi}_{p i}, \boldsymbol{\varphi}_{p j}\right)+k(k-1)\left(\operatorname{div} \boldsymbol{\varphi}_{p i}\right.$, $\left.\operatorname{div} \varphi_{p j}\right) / 2(1 \leq i, j \leq d)$.

Remark 2 Supposing that $\Im_{h}$ is a quasi consistent regular triangulation and $U_{h}$ and $\boldsymbol{W}_{h}$ are, separately, the spaces of piecewise linear polynomials and polynomial vectors, the number of whole degrees of freedom (unknowns) for Problem IV has $3 N_{h}$ ( $N_{h}$ is the number of vertices of all triangles in $\Im_{h}$ ), whereas the number of the whole degrees of freedom for Problem V only has $2 d(d \ll l \leq L \ll N)$. For scientific engineering problems in the real world, the number $N_{h}$ of vertices of all triangles in $\Im_{h}$ is more than tens of thousands, even more than a hundred million, but $d$ is only the number of the first few main eigenvalues so that it is very small (say, in Section $5, d=6$, but $N_{h}=2 \times 10^{2} \times 2 \times 10^{2}=4 \times 10^{4}$ ). Therefore, Problem V is the optimized SPDMFE extrapolation model with very few degrees of 
freedom based on POD approach for Problem I. Especially, it has no repeated computation and only employs the first few $L$ given solutions of Problem IV to obtain $n>L$ other solutions. This implies that Problem V completely differs from the majority of existing reduced-order models (see, e.g., [11-16]).

\section{Error estimates and implement procedure of algorithm}

In the following, we employ the SPDMFE approach to deduce the error estimates of solutions to Problem V and offer the implemented procedure for the optimized SPDMFE extrapolation approach.

\subsection{Error analysis of solutions to Problem V}

The results of existence, uniqueness, and stability as regards the solutions to Problem V are as follows.

Theorem 4 Under the assumptions of Theorems 1 to 3, Problem V exist only a set of solutions $\left\{\left(u_{d}^{n}, \boldsymbol{p}_{d}^{n}\right): 1 \leq n \leq N\right\} \subset U^{d} \times \boldsymbol{W}^{d}$ that meets the following stability:

$$
\left\|u_{d}^{n}\right\|_{0}+\left\|\operatorname{div} \boldsymbol{p}_{d}^{n}\right\|_{0}+\left\|\boldsymbol{p}_{d}^{n}\right\|_{0} \leq C(f), \quad 1 \leq n \leq N
$$

where $C(f)$ is a constant that is only reliant on $f$ and $T$ but not reliant on $h$ and $k$.

Proof When $n=1,2, \ldots, L$, it follows from the first and third equations that there exist a unique series of solutions $\left(u_{d}^{n}, \boldsymbol{p}_{d}^{n}\right) \in U^{d} \times \boldsymbol{W}^{d}(n=1,2, \ldots, L)$ to Problem V. By Theorem 3 and (21)-(22), we obtain

$$
\begin{aligned}
& \left\|u_{d}^{n}\right\|_{0}+\left\|\operatorname{div} \boldsymbol{p}_{d}^{n}\right\|_{0}+\left\|\boldsymbol{p}_{d}^{n}\right\|_{0} \\
& \quad=\left\|P^{d} u_{h}^{n}\right\|_{0}+\left\|\operatorname{div} \rho^{d} \boldsymbol{p}_{h}^{n}\right\|_{0}+\left\|\rho^{d} p_{h}^{n}\right\|_{0} \\
& \quad \leq\left\|u_{h}^{n}\right\|_{0}+\left\|\operatorname{div} \boldsymbol{p}_{h}^{n}\right\|_{0}+\left\|\boldsymbol{p}_{h}^{n}\right\|_{0} \leq C(f), \quad n=1,2, \ldots, L .
\end{aligned}
$$

When $L+1 \leq n \leq N$, define $a(u, v)=(u, v), F_{1}(v)=\left(2 u_{d}^{n}-u_{d}^{n-1}+k \operatorname{div} \boldsymbol{p}_{d}^{n-1} / 2-k \operatorname{div} \boldsymbol{p}_{d}^{n+1} / 2-\right.$ $\left.k^{2} \operatorname{div} \boldsymbol{p}_{d}^{n-1} / 2-k^{2} \operatorname{div} \boldsymbol{p}_{d}^{n+1} / 2+k^{2} f\left(t_{n}\right), v\right), A(\boldsymbol{p}, \boldsymbol{q})=(\boldsymbol{p}, \boldsymbol{q})+\left[k(\operatorname{div} \boldsymbol{p}, \operatorname{div} \boldsymbol{q})+k^{2}(\operatorname{div} \boldsymbol{p}, \operatorname{div} \boldsymbol{q})\right] / 2$, and $F_{2}(\boldsymbol{q})=\left(2 \boldsymbol{p}_{d}^{n}-\boldsymbol{p}_{d}^{n-1}+k \operatorname{div} \boldsymbol{p}_{d}^{n-1} / 2-k^{2} \operatorname{div} \boldsymbol{p}_{d}^{n-1} / 2+k^{2} f\left(t_{n}\right)\right.$, div $\left.\boldsymbol{q}\right)$. Then the fourth and second equations in Problem $V$ are restated as follows:

$$
\begin{aligned}
& \left\{\begin{array}{l}
A\left(\boldsymbol{p}_{d}^{n+1}, \boldsymbol{q}_{d}\right)=F_{2}(\boldsymbol{q}), \quad \forall \boldsymbol{q}_{d} \in \boldsymbol{W}^{d}, L \leq n \leq N-1, \\
\boldsymbol{p}_{d}^{L-1}=\rho^{d} \boldsymbol{p}_{h}^{L-1}, \quad \boldsymbol{p}_{d}^{L}=\rho^{d} \boldsymbol{p}_{h}^{L}, \quad(x, y) \in \Omega,
\end{array}\right. \\
& \left\{\begin{array}{l}
a\left(u_{d}^{n+1}, v_{d}\right)=F_{1}\left(v_{d}\right), \quad \forall v_{d} \in U^{d}, L \leq n \leq N-1, \\
u_{d}^{L-1}=P^{d} u_{h}^{L-1}, \quad u_{d}^{L}=P^{d} u_{h}^{L}, \quad(x, y) \in \Omega .
\end{array}\right.
\end{aligned}
$$

It is obvious that, for given $k, \boldsymbol{p}_{d}^{n-1}, \boldsymbol{p}_{d}^{n}$, and $f\left(t_{n}\right), F_{2}(\boldsymbol{q})$ is a continuous linear functional on $\boldsymbol{W}^{d}$. Since $A(\boldsymbol{p}, \boldsymbol{p})=(\boldsymbol{p}, \boldsymbol{p})+\left[k(\operatorname{div} \boldsymbol{p}, \operatorname{div} \boldsymbol{p})+k^{2}(\operatorname{div} \boldsymbol{p}, \operatorname{div} \boldsymbol{p})\right] / 2 \geq \alpha\left(\|\boldsymbol{p}\|_{0}^{2}+\|\operatorname{div} \boldsymbol{p}\|_{0}^{2}\right)($ where $\left.\alpha=\min \left\{1,\left(k+k^{2}\right) / 2\right\}\right), A(\boldsymbol{p}, \boldsymbol{q})$ on $\boldsymbol{W} \times \boldsymbol{W}$ is positive definite. And it is obvious that $A(\boldsymbol{p}, \boldsymbol{q})$ is a continuous bilinear functional on $\boldsymbol{W} \times \boldsymbol{W}$, therefore it follows by the Lax-Milgram theorem (see [21] or [22]) that for equation (29) there exist only a set of solutions $\left\{\boldsymbol{p}_{d}^{n}\right.$ : $L+1 \leq n \leq N\}$, independent of equation (30). It is obvious that $a(u, v)$ is a continuous 
positive definite bilinear functional on $U^{d} \times U^{d}$ and $F_{1}(v)$ is a continuous linear functional on $U^{d}$ for given $u_{d}^{n}, u_{d}^{n-1}, \boldsymbol{p}_{d}^{n-1}, \boldsymbol{p}_{d}^{n+1}$, and $f\left(t_{n}\right)$, too. Thus, it follows still by means of the LaxMilgram theorem (see [21] or [22]) that for equation (30) there exist only a set of solutions $\left\{u_{d}^{n}: L+1 \leq n \leq N\right\}$.

By choosing $\boldsymbol{q}_{d}=\boldsymbol{p}_{d}^{n+1}-\boldsymbol{p}_{d}^{n-1}$ in equation (29) and adopting Hölder inequality and Cauchy inequality, we have

$$
\begin{aligned}
& \left\|\boldsymbol{p}_{d}^{n+1}-\boldsymbol{p}_{d}^{n}\right\|_{0}^{2}-\left\|\boldsymbol{p}_{d}^{n}-\boldsymbol{p}_{d}^{n-1}\right\|_{0}^{2}+\frac{k}{2}\left\|\operatorname{div}\left(\boldsymbol{p}_{d}^{n+1}-\boldsymbol{p}_{d}^{n-1}\right)\right\|_{0}^{2} \\
& +\frac{k^{2}}{2}\left(\left\|\operatorname{div} \boldsymbol{p}_{d}^{n+1}\right\|_{0}^{2}-\left\|\operatorname{div} \boldsymbol{p}_{d}^{n-1}\right\|_{0}^{2}\right) \leq k^{3}\left\|f\left(t_{n}\right)\right\|_{0}^{2}+\frac{k}{4}\left\|\operatorname{div}\left(\boldsymbol{p}_{d}^{n+1}-\boldsymbol{p}_{d}^{n-1}\right)\right\|_{0}^{2}
\end{aligned}
$$

Note that if $k=O(h)$ or $k=O\left(h^{2}\right)$, it follows by Theorem 3 and Taylor's formula that $\| \boldsymbol{p}_{d}^{L}-$ $\boldsymbol{p}_{d}^{L-1} \|_{0} \leq C(f)\left(k+h^{m+1}\right) \leq C(f) k$. Simplifying (31) and then summing from $L$ to $n$ yield

$$
\begin{aligned}
& \left\|\boldsymbol{p}_{d}^{n+1}-\boldsymbol{p}_{d}^{n}\right\|_{0}^{2}+k^{2}\left\|\operatorname{div} \boldsymbol{p}_{d}^{n+1}\right\|_{0}^{2}+k^{2}\left\|\operatorname{div} \boldsymbol{p}_{d}^{n}\right\|_{0}^{2}+k \sum_{i=L}^{n}\left\|\operatorname{div}\left(\boldsymbol{p}_{d}^{i+1}-\boldsymbol{p}_{d}^{i-1}\right)\right\|_{0}^{2} \\
& \leq 4 k^{3} \sum_{i=1}^{n}\left\|f\left(t_{i}\right)\right\|_{0}^{2}+\frac{k^{2}}{2}\left\|\operatorname{div} \boldsymbol{p}_{d}^{L-1}\right\|_{0}^{2}+\frac{k^{2}}{2}\left\|\operatorname{div} \boldsymbol{p}_{d}^{L}\right\|_{0}^{2}+\left\|\boldsymbol{p}_{d}^{L}-\boldsymbol{p}_{d}^{L-1}\right\|_{0}^{2} \\
& \leq\left(4 T+\tilde{C}^{2}\right) k^{2}\|f\|_{L^{\infty}\left(L^{2}\right)}^{2}+C(f) k^{2} \leq C(f) k^{2} .
\end{aligned}
$$

Further, it follows that

$$
\left\|\operatorname{div} \boldsymbol{p}_{d}^{n}\right\|_{0} \leq C(f), \quad L+1 \leq n \leq N
$$

and

$$
\left\|\boldsymbol{p}_{d}^{n+1}\right\|_{0}-\left\|\boldsymbol{p}_{d}^{n}\right\|_{0} \leq\left\|\boldsymbol{p}_{d}^{n+1}-\boldsymbol{p}_{d}^{n}\right\|_{0} \leq C(f) k
$$

Summing from $L$ to $n-1$ for (34) and employing (28) yield

$$
\left\|\boldsymbol{p}_{d}^{n}\right\|_{0} \leq C(f) n k+\left\|\boldsymbol{p}_{d}^{L}\right\|_{0} \leq C(f), \quad L+1 \leq n \leq N .
$$

Choosing $v_{d}=u_{d}^{n+1}-u_{d}^{n}$ in (30) and employing the Hölder and Cauchy inequalities yield

$$
\begin{aligned}
\left\|u_{d}^{n+1}-u_{d}^{n}\right\|_{0}^{2} & \\
\leq & \left\|u_{d}^{n}-u_{d}^{n-1}\right\|\left\|_{0}\right\| u_{d}^{n+1}-u_{d}^{n}\left\|_{0}+\frac{k}{2}\right\| \operatorname{div}\left(\boldsymbol{p}_{d}^{n+1}-\boldsymbol{p}_{d}^{n-1}\right)\left\|_{0}\right\| u_{d}^{n+1}-u_{d}^{n} \|_{0} \\
& +\frac{k^{2}}{2}\left\|\operatorname{div}\left(\boldsymbol{p}_{d}^{n+1}+\boldsymbol{p}_{d}^{n-1}\right)\right\|_{0}\left\|u_{d}^{n+1}-u_{d}^{n}\right\|_{0}+\frac{k^{2}}{2}\left\|f\left(t_{n}\right)\right\|_{0}\left\|u_{d}^{n+1}-u_{d}^{n}\right\|_{0} .
\end{aligned}
$$

Note that if $k=O(h)$ or $k=O\left(h^{2}\right)$, it follows by Theorem 3 and Taylor's formula that $\| u_{d}^{L}-$ $u_{d}^{L-1} \|_{0} \leq C(f)\left(k+h^{m+1}\right) \leq C(f) k$. Simplifying (36) and then summing from $L$ to $n$ and 
employing (32) and (33) yield

$$
\begin{aligned}
\left\|u_{d}^{n+1}-u_{d}^{n}\right\|_{0} \leq & \left\|u_{d}^{L}-u_{d}^{L-1}\right\|_{0}+k \sum_{i=1}^{n}\left\|\operatorname{div}\left(\boldsymbol{p}_{d}^{i+1}-\boldsymbol{p}_{d}^{i-1}\right)\right\|_{0} \\
& +k^{2} \sum_{i=L}^{n}\left\|\operatorname{div}\left(\boldsymbol{p}_{d}^{i+1}+\boldsymbol{p}_{d}^{i-1}\right)\right\|_{0}+k^{2} \sum_{L=1}^{n}\left\|f\left(t_{i}\right)\right\|_{0} \leq C(f) k .
\end{aligned}
$$

Applying the triangle inequality to (37) yields

$$
\left\|u_{d}^{n+1}\right\|_{0}-\left\|u_{d}^{n}\right\|_{0} \leq C(f) k
$$

Summing from $L$ to $n-1$ for (38) and employing (28) yields

$$
\left\|u_{d}^{n}\right\|_{0} \leq\left\|u_{d}^{L}\right\|_{0}+C(f) \leq C(f)
$$

Combining (39) with (28), (33), and (35) yields (27), which accomplishes the demonstration of Theorem 4.

For the solutions for Problem V, we have the following error estimates.

Theorem 5 Under the assumptions of Theorems 1 to 4 , if $f \in W^{1, \infty}\left(0, T ; H^{m}(\Omega)\right)$, then the errors between the solution $u(t)$ to Problem I and the solutions $u_{h}^{n}$ to Problem V satisfy the following estimate formulas:

$$
\begin{aligned}
& \left\|u\left(t_{n}\right)-u_{d}^{n}\right\|_{0}+\left\|\boldsymbol{p}\left(t_{n}\right)-\boldsymbol{p}_{d}^{n}\right\|_{0} \\
& \leq C\left(k^{2}+h^{m+1}\right)+C \sqrt{L}\left(k \sum_{i=d+1}^{l} \lambda_{j}\right)^{1 / 2}, \quad 1 \leq n \leq L ; \\
& \left\|u\left(t_{n}\right)-u_{d}^{n}\right\|_{0}+\left\|\boldsymbol{p}\left(t_{n}\right)-\boldsymbol{p}_{d}^{n}\right\|_{0}+\left\|\operatorname{div}\left(\boldsymbol{p}\left(t_{n}\right)-\boldsymbol{p}_{d}^{n}\right)\right\|_{0} \\
& \quad \leq C\left(k^{2}+h^{m+1}\right)+C \sqrt{L}\left(k \sum_{i=d+1}^{l} \lambda_{j}\right)^{1 / 2}, \quad L+1 \leq n \leq N .
\end{aligned}
$$

Proof When $1 \leq n \leq L$, it follows from Lemma 1 that

$$
\left\|u_{n}-u_{d}^{n}\right\|_{0}+\left\|\boldsymbol{p}_{n}-\boldsymbol{p}_{d}^{n}\right\|_{0} \leq C \sqrt{L}\left(k \sum_{i=d+1}^{l} \lambda_{j}\right)^{1 / 2}, \quad 1 \leq n \leq L .
$$

It follows (40) from (42) and Theorem 3.

When $L \leq n \leq N$, let $e^{n}=u_{h}^{n}-u_{d}^{n}$ and $\boldsymbol{E}^{n}=\boldsymbol{p}_{h}^{n}-\boldsymbol{p}_{d}^{n}$. Choosing $v_{h}=v_{d}^{n}$ and $\boldsymbol{q}_{h}=\boldsymbol{q}_{d}$ in Problem IV yields the following error equations:

$$
\begin{aligned}
& \left(\boldsymbol{E}^{n+1}-2 \boldsymbol{E}^{n}+\boldsymbol{E}^{n-1}, \boldsymbol{q}_{d}\right)+\frac{k}{2}\left(\operatorname{div} \boldsymbol{E}^{n+1}-\operatorname{div} \boldsymbol{E}^{n-1}, \operatorname{div} \boldsymbol{q}_{d}\right) \\
& +\frac{k^{2}}{2}\left(\operatorname{div} \boldsymbol{E}^{n+1}+\operatorname{div} \boldsymbol{E}^{n-1}, \operatorname{div} \boldsymbol{q}_{d}\right)=0, \quad \forall \boldsymbol{q}_{d} \in \boldsymbol{W}^{d}, L \leq n \leq N-1 ;
\end{aligned}
$$




$$
\begin{aligned}
& \left(e^{n+1}-2 e^{n}+e^{n-1}, v_{d}\right)+\frac{k}{2}\left(\operatorname{div} \boldsymbol{E}^{n+1}-\operatorname{div} \boldsymbol{E}^{n-1}, v_{d}\right) \\
& \quad+k^{2}\left(\operatorname{div} \boldsymbol{E}^{n+1}+\operatorname{div} \boldsymbol{E}^{n-1}, v_{d}\right)=0, \quad \forall \in v_{d} \in U^{d}, L \leq n \leq N-1 .
\end{aligned}
$$

Let $\boldsymbol{\sigma}^{n}=\boldsymbol{p}_{h}^{n}-\rho^{d} \boldsymbol{p}_{h}^{n}$ and $\boldsymbol{\theta}^{n}=\rho^{d} \boldsymbol{p}_{h}^{n}-\boldsymbol{p}_{d}^{n}$. It follows by (43), (19), and the Hölder and Cauchy inequalities that

$$
\begin{aligned}
\| \boldsymbol{\theta}^{n+1} & -\boldsymbol{\theta}^{n}\left\|_{0}^{2}-\right\| \boldsymbol{\theta}^{n}-\boldsymbol{\theta}^{n-1}\left\|_{0}^{2}+\frac{k}{2}\right\| \operatorname{div}\left(\boldsymbol{\theta}^{n+1}-\boldsymbol{\theta}^{n-1}\right) \|_{0}^{2} \\
& +\frac{k^{2}}{2}\left(\left\|\operatorname{div} \boldsymbol{\theta}^{n+1}\right\|_{0}^{2}-\left\|\operatorname{div} \boldsymbol{\theta}^{n-1}\right\|_{0}^{2}\right) \\
= & \left(\boldsymbol{\theta}^{n+1}-2 \boldsymbol{\theta}^{n}+\boldsymbol{\theta}^{n-1}, \boldsymbol{\theta}^{n+1}-\boldsymbol{\theta}^{n-1}\right)+\frac{k}{2}\left(\operatorname{div}\left(\boldsymbol{\theta}^{n+1}-\boldsymbol{\theta}^{n-1}\right), \operatorname{div}\left(\boldsymbol{\theta}^{n+1}-\boldsymbol{\theta}^{n-1}\right)\right) \\
& +\frac{k^{2}}{2}\left(\operatorname{div}\left(\boldsymbol{\theta}^{n+1}+\boldsymbol{\theta}^{n-1}\right), \operatorname{div}\left(\boldsymbol{\theta}^{n+1}-\boldsymbol{\theta}^{n-1}\right)\right) \\
= & \left(\boldsymbol{E}^{n+1}-2 \boldsymbol{E}^{n}+\boldsymbol{E}^{n-1}, \boldsymbol{\theta}^{n+1}-\boldsymbol{\theta}^{n-1}\right)+\frac{k}{2}\left(\operatorname{div}\left(\boldsymbol{E}^{n+1}-\boldsymbol{E}^{n-1}\right), \operatorname{div}\left(\boldsymbol{\theta}^{n+1}-\boldsymbol{\theta}^{n-1}\right)\right) \\
& +\frac{k^{2}}{2}\left(\operatorname{div}\left(\boldsymbol{E}^{n+1}+\boldsymbol{E}^{n-1}\right), \operatorname{div}\left(\boldsymbol{\theta}^{n+1}-\boldsymbol{\theta}^{n-1}\right)\right)-\left(\boldsymbol{\sigma}^{n+1}-2 \boldsymbol{\sigma}^{n}+\boldsymbol{\sigma}^{n-1}, \boldsymbol{\theta}^{n+1}-\boldsymbol{\theta}^{n-1}\right) \\
& -\frac{k}{2}\left(\operatorname{div}\left(\boldsymbol{\sigma}^{n+1}-\boldsymbol{\sigma}^{n-1}\right), \operatorname{div}\left(\boldsymbol{\theta}^{n+1}-\boldsymbol{\theta}^{n-1}\right)\right)-\frac{k^{2}}{2}\left(\operatorname{div}\left(\boldsymbol{\sigma}^{n+1}+\boldsymbol{\sigma}^{n-1}\right), \operatorname{div}\left(\boldsymbol{\theta}^{n+1}-\boldsymbol{\theta}^{n-1}\right)\right) \\
= & -\left(\boldsymbol{\sigma}^{n+1}-2 \boldsymbol{\sigma}^{n}+\boldsymbol{\sigma}^{n-1}, \boldsymbol{\theta}^{n+1}-\boldsymbol{\theta}^{n-1}\right)+\frac{k}{2}\left(\boldsymbol{\sigma}^{n+1}-\boldsymbol{\sigma}^{n-1}, \boldsymbol{\theta}^{n+1}-\boldsymbol{\theta}^{n-1}\right) \\
& +\frac{k^{2}}{2}\left(\boldsymbol{\sigma}^{n+1}+\boldsymbol{\sigma}^{n-1}, \boldsymbol{\theta}^{n+1}-\boldsymbol{\theta}^{n-1}\right) \\
\leq & \left(\left\|\boldsymbol{\sigma}^{n+1}-2 \boldsymbol{\sigma}^{n}+\boldsymbol{\sigma}^{n-1}\right\|_{0}\right)\left\|\boldsymbol{\theta}^{n+1}-\boldsymbol{\theta}^{n-1}\right\|_{0} \\
& +\frac{k}{2}\left(\left\|\boldsymbol{\sigma}^{n+1}-\boldsymbol{\sigma}^{n-1}\right\|_{0}\right)\left\|\boldsymbol{\theta}^{n+1}-\boldsymbol{\theta}^{n-1}\right\|_{0} \\
& +\frac{k^{2}}{2}\left(\left\|\boldsymbol{\sigma}^{n+1}\right\|_{0}+\left\|\boldsymbol{\sigma}^{n-1}\right\|_{0}\right)\left\|\boldsymbol{\theta}^{n+1}-\boldsymbol{\theta}^{n-1}\right\|_{0} .
\end{aligned}
$$

Note that $\left\|\boldsymbol{\theta}^{n+1}-\boldsymbol{\theta}^{n-1}\right\|_{0} \leq\left\|\boldsymbol{\theta}^{n+1}-\boldsymbol{\theta}^{n}\right\|_{0}+\left\|\boldsymbol{\theta}^{n}-\boldsymbol{\theta}^{n-1}\right\|_{0}$ and $\left\|\boldsymbol{\theta}^{n+1}-\boldsymbol{\theta}^{n-1}\right\|_{0} \leq\left\|\boldsymbol{\theta}^{n+1}\right\|_{0}+$ $\left\|\boldsymbol{\theta}^{n-1}\right\|_{0} \leq C\left(\left\|\operatorname{div} \boldsymbol{\theta}^{n+1}\right\|_{0}+\left\|\operatorname{div} \boldsymbol{\theta}^{n-1}\right\|_{0}\right)$. We have from Taylor's formula and Lemma 1 $\left\|\boldsymbol{\sigma}^{n+1}-2 \boldsymbol{\sigma}^{n}+\boldsymbol{\sigma}^{n-1}\right\|_{0} \leq C\left(k^{4}+k^{2} h^{m+1}\right)$ and $\left\|\boldsymbol{\sigma}^{n+1}-\boldsymbol{\sigma}^{n-1}\right\|_{0} \leq C\left(k^{3}+k h^{m+1}\right)$. Thus, simplifying (45) yields

$$
\begin{gathered}
\left\|\boldsymbol{\theta}^{n+1}-\boldsymbol{\theta}^{n}\right\|_{0}-\left\|\boldsymbol{\theta}^{n}-\boldsymbol{\theta}^{n-1}\right\|_{0}+\frac{k}{2}\left(\left\|\operatorname{div} \boldsymbol{\theta}^{n+1}\right\|_{0}-\left\|\operatorname{div} \boldsymbol{\theta}^{n-1}\right\|_{0}\right) \\
+\frac{k^{2}}{2}\left(\left\|\operatorname{div} \boldsymbol{\theta}^{n+1}\right\|_{0}-\left\|\operatorname{div} \boldsymbol{\theta}^{n-1}\right\|_{0}\right) \\
\leq\left\|\boldsymbol{\theta}^{n+1}-\boldsymbol{\theta}^{n}\right\|_{0}-\left\|\boldsymbol{\theta}^{n}-\boldsymbol{\theta}^{n-1}\right\|_{0}+\frac{k}{2}\left\|\operatorname{div} \boldsymbol{\theta}^{n+1}-\operatorname{div} \boldsymbol{\theta}^{n-1}\right\|_{0} \\
+\frac{k^{2}}{2}\left(\left\|\operatorname{div} \boldsymbol{\theta}^{n+1}\right\|_{0}-\left\|\operatorname{div} \boldsymbol{\theta}^{n-1}\right\|_{0}\right) \leq C k^{2}\left(h^{m+1}+k^{2}\right) .
\end{gathered}
$$


If $k \leq 1$, summing from $L$ to $n$ for (46) and employing Lemma 1 and (42) yield

$$
\begin{gathered}
\left\|\boldsymbol{\theta}^{n+1}-\boldsymbol{\theta}^{n}\right\|_{0}+\frac{k}{2}\left(\left\|\operatorname{div} \boldsymbol{\theta}^{n+1}\right\|_{0}+\left\|\operatorname{div} \boldsymbol{\theta}^{n}\right\|_{0}\right)+\frac{k^{2}}{2}\left\|\operatorname{div} \boldsymbol{\theta}^{n+1}\right\|_{0} \\
\leq k\left(\left\|\operatorname{div} \boldsymbol{\theta}^{L}\right\|_{0}+\left\|\operatorname{div} \boldsymbol{\theta}^{L-1}\right\|_{0}\right)+C(n-L) k^{2}\left(h^{m+1}+k^{2}\right) \\
\leq C(n-L) k^{2}\left(h^{m+1}+k^{2}\right)+C k \sqrt{L}\left(k \sum_{i=d+1}^{l} \lambda_{j}\right)^{1 / 2} .
\end{gathered}
$$

Applying the triangle inequality to (47) yields

$$
\left\|\boldsymbol{\theta}^{n+1}\right\|_{0}-\left\|\boldsymbol{\theta}^{n}\right\|_{0} \leq C(n-L) k^{2}\left(h^{m+1}+k^{2}\right)+C k \sqrt{L}\left(k \sum_{i=d+1}^{l} \lambda_{j}\right)^{1 / 2} .
$$

Summing from $L$ to $n-1$ for (48) and employing Lemma 1 and (42) yield

$$
\begin{aligned}
\left\|\boldsymbol{\theta}^{n+1}\right\|_{0} & \leq\left\|\boldsymbol{\theta}^{L}\right\|_{0}+C(n-L)^{2} k^{2}\left(h^{m+1}+k^{2}\right)+C k(n-L-1) \sqrt{L}\left(k \sum_{i=d+1}^{l} \lambda_{j}\right)^{1 / 2} \\
& \leq C\left(h^{m+1}+k^{2}\right)+C k(n-L) \sqrt{L}\left(k \sum_{i=d+1}^{l} \lambda_{j}\right)^{1 / 2} .
\end{aligned}
$$

It follows from (47) and (49) that

$$
\left\|\boldsymbol{\theta}^{n}\right\|_{0}+\left\|\operatorname{div} \boldsymbol{\theta}^{n}\right\|_{0} \leq C\left(h^{m+1}+k^{2}\right)+C \sqrt{L}\left(k \sum_{i=d+1}^{l} \lambda_{j}\right)^{1 / 2} .
$$

Let $\varpi^{n}=u_{h}^{n}-P^{d} u_{h}^{n}$ and $\omega^{n}=P^{d} u_{h}^{n}-u_{d}^{n}$. By (18) and (44), we obtain

$$
\begin{aligned}
& \left(\omega^{n+1}-2 \omega^{n}+\omega^{n-1}, \omega^{n+1}-\omega^{n}\right) \\
& \quad=\left(e^{n+1}-2 e^{n}+e^{n-1}, \omega^{n+1}-\omega^{n}\right)-\left(\varpi^{n+1}-2 \varpi^{n}+\varpi^{n-1}, \omega^{n+1}-\omega^{n}\right) \\
& \quad=-\frac{k}{2}\left(\operatorname{div}\left(\boldsymbol{E}^{n+1}-\boldsymbol{E}^{n-1}\right), \omega^{n+1}-\omega^{n}\right)-\frac{k^{2}}{2}\left(\operatorname{div}\left(\boldsymbol{E}^{n+1}+\boldsymbol{E}^{n-1}\right), \omega^{n+1}-\omega^{n}\right) .
\end{aligned}
$$

Applying the Hölder and Cauchy inequalities to (51) yields

$$
\begin{aligned}
\left\|\omega^{n+1}-\omega^{n}\right\|_{0}^{2} \leq & \left\|\omega^{n}-\omega^{n-1}\right\|_{0}\left\|\omega^{n+1}-\omega^{n}\right\|_{0} \\
& +\frac{k}{2}\left\|\operatorname{div}\left(\boldsymbol{E}^{n+1}-\boldsymbol{E}^{n-1}\right)\right\|_{0}\left\|\omega^{n+1}-\omega^{n}\right\|_{0} \\
& +\frac{k^{2}}{2}\left\|\operatorname{div}\left(\boldsymbol{E}^{n+1}+\boldsymbol{E}^{n-1}\right)\right\|_{0}\left\|\omega^{n+1}-\omega^{n}\right\|_{0}
\end{aligned}
$$


Simplifying (52) and then summing from $L$ to $n$ yield

$$
\begin{aligned}
\left\|\omega^{n+1}-\omega^{n}\right\|_{0} \leq & k \sum_{i=1}^{n}\left\|\operatorname{div}\left(\boldsymbol{\sigma}^{i+1}-\boldsymbol{\sigma}^{i-1}\right)\right\|_{0}+k^{2} \sum_{i=1}^{n+1}\left\|\operatorname{div}\left(\boldsymbol{\sigma}^{i+1}+\boldsymbol{\sigma}^{i-1}\right)\right\|_{0} \\
& +k \sum_{i=1}^{n}\left\|\operatorname{div}\left(\boldsymbol{\theta}^{i+1}-\boldsymbol{\theta}^{i-1}\right)\right\|_{0}+k^{2} \sum_{i=1}^{n+1}\left\|\operatorname{div}\left(\boldsymbol{\theta}^{i+1}+\boldsymbol{\theta}^{i-1}\right)\right\|_{0} .
\end{aligned}
$$

Note that it follows from Taylor's formula and Lemma 1 that $\left\|\operatorname{div}\left(\sigma^{i+1}-\sigma^{i-1}\right)\right\|_{0} \leq C k^{3}+$ $C k h^{m+1}$ and summing from $L$ to $n$ for (46) yields $k \sum_{i=1}^{n}\left\|\operatorname{div}\left(\boldsymbol{\theta}^{i+1}-\boldsymbol{\theta}^{i-1}\right)\right\|_{0} \leq C k\left(h^{m+1}+k^{2}\right)$. Thus, it follows from (50) and (53) that

$$
\left\|\omega^{n+1}-\omega^{n}\right\|_{0} \leq C k\left(h^{m+1}+k^{2}\right)+C k \sqrt{L}\left(k \sum_{i=d+1}^{l} \lambda_{j}\right)^{1 / 2} .
$$

Applying the triangle inequality to (54) yields

$$
\left\|\omega^{n+1}\right\|_{0}-\left\|\omega^{n}\right\|_{0} \leq C k\left(h^{m+1}+k^{2}\right)+C k \sqrt{L}\left(k \sum_{i=d+1}^{l} \lambda_{j}\right)^{1 / 2} .
$$

Summing from $L$ to $n-1$ for (55) and employing Lemma 1 and (42) yield

$$
\begin{aligned}
\left\|\omega^{n}\right\|_{0} & \leq\left\|\omega^{L}\right\|_{0}+C n k\left(h^{m+1}+k^{2}\right)+C n k \sqrt{L}\left(k \sum_{i=d+1}^{l} \lambda_{j}\right)^{1 / 2} \\
& \leq C\left(h^{m+1}+k^{2}\right)+C \sqrt{L}\left(k \sum_{i=d+1}^{l} \lambda_{j}\right)^{1 / 2} .
\end{aligned}
$$

Combining (50) with (56), Lemma 1, and Theorem 3 yields (41), which accomplishes the proof of Theorem 5 .

Remark 3 The error formulas in Theorem 5 express that $L$ cannot be too large so that $\sqrt{L}<5$ (usually taken as $L=20$ ). In this case, if $m=1$ and $h=O(k)$, the error $k^{2}+\sqrt{L}\left(k \sum_{i=d+1}^{l} \lambda_{j}\right)^{1 / 2}$ is optimized, it offers the suggestions to determine the number $d$ of POD bases and the number $L$ of the snapshots, i.e., as long as choosing $L$ that meets $\sqrt{L}<5$ and $\left(k \sum_{i=d+1}^{l} \lambda_{j}\right)^{1 / 2}=O\left(k^{2}\right)$, then it is theoretically ensured that the solutions to Problem $\mathrm{V}$ have the $k^{2}$-order convergent accuracy.

\subsection{The implement procedure of the optimized SPDMFE extrapolation approach}

The implement procedure of the optimized SPDMFE extrapolation approach includes the following seven steps. 
Step 1 For the given $\varepsilon, \gamma, \psi(x, y, t), \psi_{0}(x, y), \psi_{1}(x, y), f(x, y, t), k$, and $h$ meeting $k=O(h)$, by solving the following classical SPDMFE model at the first $L(\sqrt{L}<5)$ steps:

$$
\left\{\begin{array}{l}
\left(u_{h}^{0}, v_{h}\right)=\left(\psi_{0}(x, y), v_{h}\right), \quad \forall v_{h} \in V_{h} ; \quad\left(u_{h}^{1}, v_{h}\right)=\left(\psi_{1}(x, y), v_{h}\right), \quad \forall v_{h} \in V_{h} ; \\
\left(\boldsymbol{p}_{h}^{0}, \boldsymbol{q}_{h}\right)=\left(\nabla \psi_{0}(x, y), \boldsymbol{q}_{h}\right), \quad \forall \boldsymbol{q}_{h} \in \boldsymbol{W}_{h} ; \quad\left(\boldsymbol{p}_{h}^{1}, \boldsymbol{q}_{h}\right)=\left(\nabla \psi_{1}(x, y), \boldsymbol{q}_{h}\right), \quad \forall \boldsymbol{q}_{h} \in \boldsymbol{W}_{h} ; \\
\left(\bar{\partial}_{t} \bar{\partial}_{t} u_{h}^{n}, v_{h}\right)+\varepsilon\left(\operatorname{div}\left(\bar{\partial}_{t} \boldsymbol{p}_{h}^{n}+\bar{\partial}_{t} \boldsymbol{p}_{h}^{n-1}\right), v_{h}\right)+\gamma\left(\operatorname{div} \boldsymbol{p}_{h}^{n, \frac{1}{2}}, v_{h}\right)=\left(f\left(t_{h}\right), v_{h}\right), \quad \forall v_{h} \in U_{h}, \\
\left(\bar{\partial}_{t} \bar{\partial}_{t} \boldsymbol{p}_{h}^{n}, \boldsymbol{q}_{h}\right)+\varepsilon\left(\operatorname{div}\left(\bar{\partial}_{t} \boldsymbol{p}_{h}^{n}+\bar{\partial}_{t} \boldsymbol{p}_{h}^{n-1}\right), \operatorname{div} \boldsymbol{q}_{h}\right)+\gamma\left(\operatorname{div} \boldsymbol{p}_{h}^{n, \frac{1}{2}}, \operatorname{div} \boldsymbol{q}_{h}\right) \\
\quad=\left(f\left(t_{n}\right), \operatorname{div} \boldsymbol{q}_{h}\right), \quad \forall \boldsymbol{q}_{h} \in \boldsymbol{W}_{h}, n=1,2, \ldots, L
\end{array}\right.
$$

we obtain the snapshots $\boldsymbol{V}_{i}=\left(u_{h}^{i}, \boldsymbol{p}_{h}^{i}\right)(1 \leq i \leq L)$.

Step 2 Compile the correlation matrix $\boldsymbol{A}=\left(A_{i j}\right)_{L \times L}$, where $A_{i j}=\left[\left(u_{h}^{i}, u_{h}^{j}\right)+\left(\boldsymbol{p}_{h}^{i}, \boldsymbol{p}_{h}^{j}\right)+\right.$ $\left.\left(\operatorname{div} \boldsymbol{p}_{h}^{i}, \operatorname{div} \boldsymbol{p}_{h}^{j}\right)\right] / L$.

Step 3 Find the eigenvalues $\lambda_{1} \geq \lambda_{2} \geq \cdots \geq \lambda_{l}>0\left(l=\operatorname{dim}\left\{\left(u_{h}^{n}, \boldsymbol{p}_{h}^{n}\right): 1 \leq n \leq L\right\}\right)$ and associated with eigenvectors $\boldsymbol{v}^{j}=\left(a_{1}^{j}, a_{2}^{j}, \ldots, a_{L}^{j}\right)^{T}(1 \leq j \leq l)$ of the matrix $\boldsymbol{A}$.

Step 4 Determine the number $d$ of POD bases that meets $\sum_{j=d+1}^{l} \lambda_{j} \leq k^{3}$.

Step 5 Obtain the POD bases $\left(\varphi_{u j}, \boldsymbol{\varphi}_{p j}\right)=\sum_{i=1}^{L} a_{i}^{j}\left(u_{h}^{i}, \boldsymbol{p}_{h}^{i}\right) / \sqrt{L \lambda_{j}}(1 \leq j \leq d)$.

Step 6 By settling the following optimized SPDMFE extrapolation model which only has $2 d$ degrees of freedom at each time level

$$
\begin{aligned}
& \left\{\begin{array}{l}
\beta_{j}^{n}=\left(\boldsymbol{p}_{h}^{n}, \boldsymbol{\varphi}_{p j}\right)+\left(\operatorname{div} \boldsymbol{p}_{h}^{n}, \operatorname{div} \boldsymbol{\varphi}_{p j}\right), \quad 1 \leq j \leq d, 1 \leq n \leq L ; \\
a_{i j}=\left(\boldsymbol{\varphi}_{p i}, \boldsymbol{\varphi}_{p j}\right)+0.5 k(k \gamma+\varepsilon)\left(\operatorname{div} \boldsymbol{\varphi}_{p i}, \operatorname{div} \boldsymbol{\varphi}_{p j}\right), \\
b_{i j}=\left(\boldsymbol{\varphi}_{p i}, \boldsymbol{\varphi}_{p j}\right)+0.5 k(k \gamma-\varepsilon)\left(\operatorname{div} \boldsymbol{\varphi}_{p i}, \operatorname{div} \boldsymbol{\varphi}_{p j}\right), \quad 1 \leq i, j \leq d, \\
\sum_{i=1}^{d} \beta_{i}^{n+1} a_{i j}=2 \sum_{i=1}^{d} \beta_{i}^{n}\left(\boldsymbol{\varphi}_{p i}, \boldsymbol{\varphi}_{p j}\right)-\sum_{i=1}^{d} \beta_{i}^{n-1} b_{i j}+\left(f\left(t_{n}\right), \operatorname{div} \boldsymbol{\varphi}_{p j}\right), \\
1 \leq j \leq d, L \leq n \leq N-1 ;
\end{array}\right. \\
& \left\{\begin{array}{c}
\alpha_{j}^{n}=\left(u_{h}^{n}, \varphi_{u j}\right), \quad 1 \leq j \leq d, 1 \leq n \leq L ; \\
\alpha_{j}^{n+1}=2 \alpha_{j}^{n}-\alpha_{j}^{n-1}+\left(f\left(t_{n}\right), \varphi_{u j}\right) \\
\quad 0.5 \sum_{i=1}^{d} k\left[(\varepsilon-k \gamma) \beta_{i}^{n-1}-(k+\varepsilon) \beta_{i}^{n+1}\right]\left(\operatorname{div} \boldsymbol{\varphi}_{p i}, \varphi_{u j}\right), \\
1 \leq j \leq d, L \leq n \leq N-1,
\end{array}\right.
\end{aligned}
$$

we obtain $\left(\alpha_{1}^{n}, \alpha_{2}^{n}, \ldots, \alpha_{d}^{n}, \beta_{1}^{n}, \beta_{2}^{n}, \ldots, \beta_{d}^{n}\right)^{T} \in \mathbf{R}^{2 d}(1 \leq n \leq N)$. Further, we obtain the optimized SPDMFE extrapolation solutions $\left(u_{d}^{n}, \boldsymbol{p}_{d}^{n}\right)=\left(\alpha_{1}^{n} \varphi_{u 1}+\alpha_{2}^{n} \varphi_{u 2}+\cdots+\alpha_{d}^{n} \varphi_{u d}, \beta_{1}^{n} \boldsymbol{\varphi}_{p 1}+\right.$ $\left.\beta_{2}^{n} \boldsymbol{\varphi}_{p 2}+\cdots+\beta_{d}^{n} \boldsymbol{\varphi}_{p d}\right)(1 \leq n \leq N)$.

Step 7 If $\left\|\left(u_{d}^{n-1}, \boldsymbol{p}_{d}^{n-1}\right)-\left(u_{d}^{n}, \boldsymbol{p}_{d}^{n}\right)\right\|_{U \times \boldsymbol{w}} \geq\left\|\left(u_{d}^{n}, \boldsymbol{p}_{d}^{n}\right)-\left(u_{d}^{n+1}, \boldsymbol{p}_{d}^{n+1}\right)\right\|_{U \times \boldsymbol{W}}(L \leq n \leq N-1)$, then $\left(u_{d}^{n}, \boldsymbol{p}_{d}^{n}\right)(1 \leq n \leq N)$ are the solutions for Problem V satisfying the desirable accuracy. Else, namely, if $\left\|\left(u_{d}^{n-1}, \boldsymbol{p}_{d}^{n-1}\right)-\left(u_{d}^{n}, \boldsymbol{p}_{d}^{n}\right)\right\|_{U \times \boldsymbol{W}}<\left\|\left(u_{d}^{n}, \boldsymbol{p}_{d}^{n}\right)-\left(u_{d}^{n+1}, \boldsymbol{p}_{d}^{n+1}\right)\right\|_{U \times \boldsymbol{W}}(L \leq n \leq N-1)$, let $\boldsymbol{V}_{i}=\left(u_{d}^{i}, \boldsymbol{p}_{d}^{i}\right)(i=n-L-1, n-L-2, \ldots, n-1)$ and return to Step 2.

Remark 4 Though Problem V is theoretically ensured with $k^{2}$-order accuracy (if $k=$ $O(h))$, due to the truncated error accumulation in the computing process, the computational accuracy exceeds the real requirement. Therefore, in order to obtain the desired 


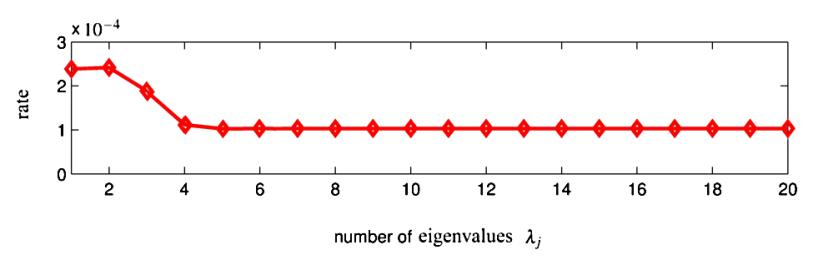

Figure 1 The change rate of eigenvalues $\lambda_{j}(j=1,2, \ldots, 20)$.

accurate numerical solutions, it is best to add Step 7, namely if the computing accuracy is unsatisfied, the desired accurate numerical solutions are obtained by renewing the snapshots and the POD bases.

\section{Some numerical simulations}

In the following, we offer some numerical simulations for confirming that the numerical conclusions are accordant with theoretical ones and validating the feasibility and efficiency of the optimized SPDMFE extrapolation approach for finding numerical solutions of 2DVWE.

We chose an irregular computational domain as follows: $\bar{\Omega}=([0,2] \times[0,2]) \cup([0.65$, $1.3] \times[2,2.03]) \mathrm{cm}^{2}$, set $f(x, y, t)=0$ and $\psi(x, y, t)=\psi_{0}(x, y)=\psi_{1}(x, y)$ that satisfy, for $0 \leq$ $t \leq T$,

$$
\psi(x, y, t)=\psi_{0}(x, y)=\psi_{1}(x, y)= \begin{cases}2-x, & \text { if }(x, y) \in[1.5,2] \times[2,2] \\ 0.5, & \text { if }(x, y) \in[0.65,1.5] \times[2,2.03] \\ 0.0, & \text { others }\end{cases}
$$

Thus, $\psi_{0}(x, y)$ and $\psi_{1}(x, y)$ all were almost everywhere derivable on $\bar{\Omega}$ and their first-order partial derivatives were almost everywhere zero on $\bar{\Omega}$. Therefore, we defined $\boldsymbol{p}(x, y, 0)=$ $\boldsymbol{p}_{t}(x, y, 0)=\nabla \psi_{0}(x, y)=\mathbf{0}$ in $\Omega$.

We first partitioned the field $\bar{\Omega}$ into $200 \times 200$ squares with edge length $\Delta x=\Delta y=10^{-2}$. Next, we linked the diagonal of the square in the same direction, partitioning each square into two triangles. Finally, by locally refining meshes so that the scale of the meshes on $[0.65,1.3] \times[2,2.03]$ and nearby $(x, 2)(0 \leq x \leq 2)$ were one-third of the meshes nearby $(x, 0)(0 \leq x \leq 2)$, we obtained the triangularization $\Im_{h}$. Thus, $h=\sqrt{2} \times 10^{-2}$. We chose the time step as $k=10^{-2}$ so that $k=O(h)$ is satisfied. We chose the MFE spaces $U_{h}$ and $\boldsymbol{W}_{h}$ as the piecewise linear polynomials and polynomial vectors, separately.

First, we found the first 20 solutions $\left(u_{h}^{n}, p_{1 h}^{n}, p_{2 h}^{n}\right)(1 \leq n \leq 20)$ for Problem IV as the snapshots and computed the eigenvalues and eigenvectors, where the change rate of eigenvalues is expressed in Figure 1. When $d=6$ and $k=10^{-2}$, we obtained $\left(k^{1 / 2} \sum_{j=6}^{20} \lambda_{j}\right)^{1 / 2} \leq$ $4 \times 10^{-4}$, which implied that we only needed to take five POD bases and this was also accordant with the change rate of eigenvalues. Thus, the optimized SPDMFE extrapolation approach (Problem V) at each time level included only $2 \times 5=10$ degrees of freedom, whereas the classical SPDMFE formulation (Problem IV) contained more than $3 \times 4 \times 10^{4}$ degrees of freedom. Therefore, the optimized SPDMFE extrapolation approach (Problem V) could not only lessen the calculation load and spare the operation time in the computing course, but it could also alleviate the truncated error accumulation. When we solved the optimized SPDMFE extrapolation approach (Problem V) including five POD 


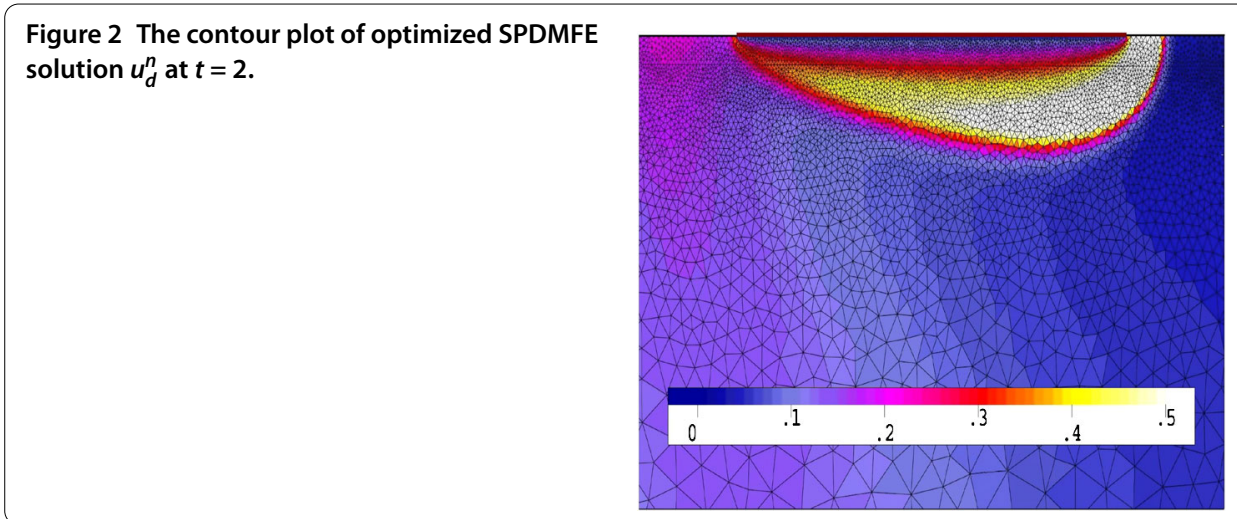

Figure 3 The contour plot of classical SPDMFE solution $u_{h}^{n}$ at $t=2$.
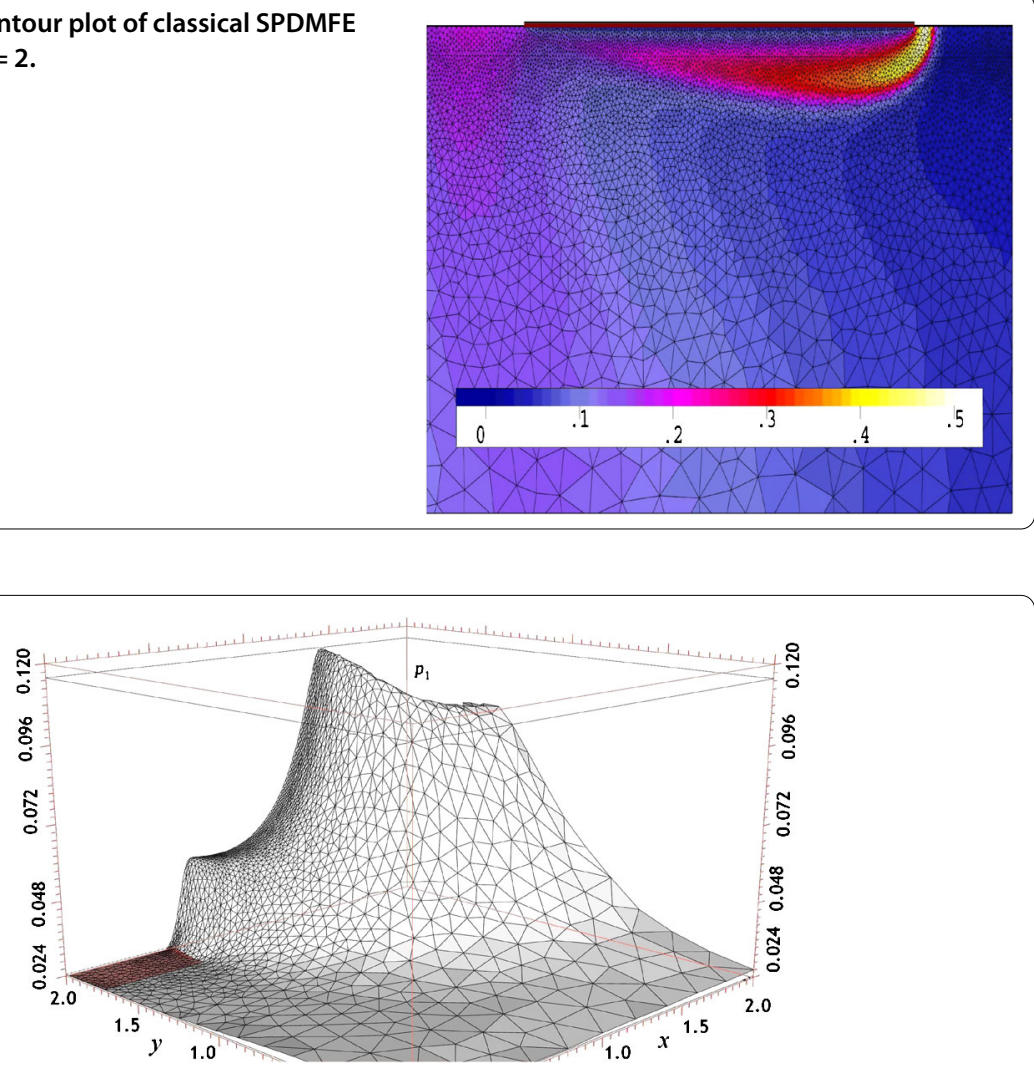

Figure 4 The 3D image of optimized SPDMFE solution $p_{1 d}^{n}$ at $t=2$.

bases, according to the seven steps of the implement procedure of the optimized SPDMFE extrapolation approach in Section 4.2, we found that the optimized SPDMFE extrapolation approach at $t=2$ still converges without updating the POD bases. The optimized solution obtained from the optimized SPDMFE extrapolation approach (Problem V) is depicted graphically in Figures 2, 4, and 6, separately. We found the numerical solutions $u_{h}^{n}$ and $\boldsymbol{p}_{h}^{n} \equiv\left(p_{1 h}^{n}, p_{2 h}^{n}\right)$ by means of the classical SPDMFE method (Problem IV) when $t=2$, depicted graphically in Figures 3, 5, and 7, separately. The charts in Figures 2, 4, and 6 are very similar to those in Figures 3, 5, and 7, separately, but the optimized SPDMFE extrapolation solutions were computed with higher efficiency than the classical SPDMFE solution 


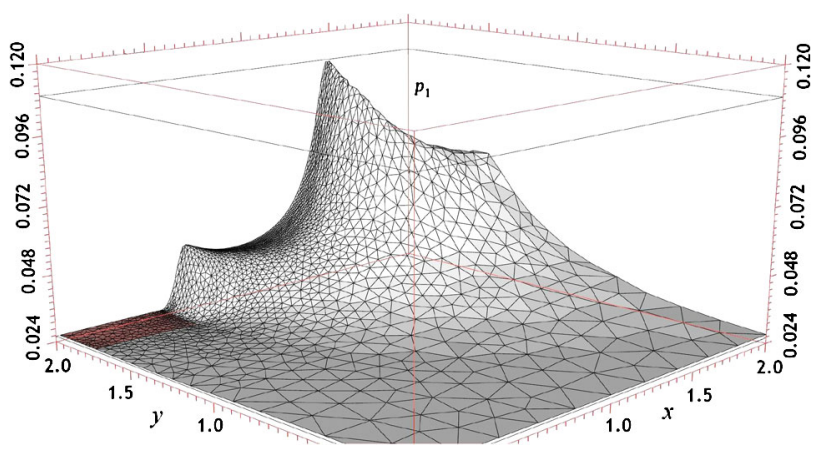

Figure 5 The 3D image of classical SPDMFE solution $p_{1 d}^{n}$ at $t=2$.

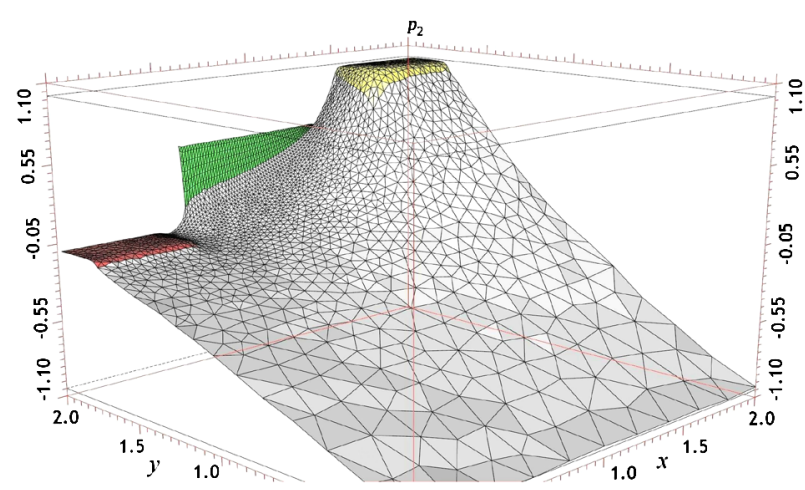

Figure 6 The 3D image of optimized SPDMFE solution $p_{2 d}^{n}$ at $t=2$.

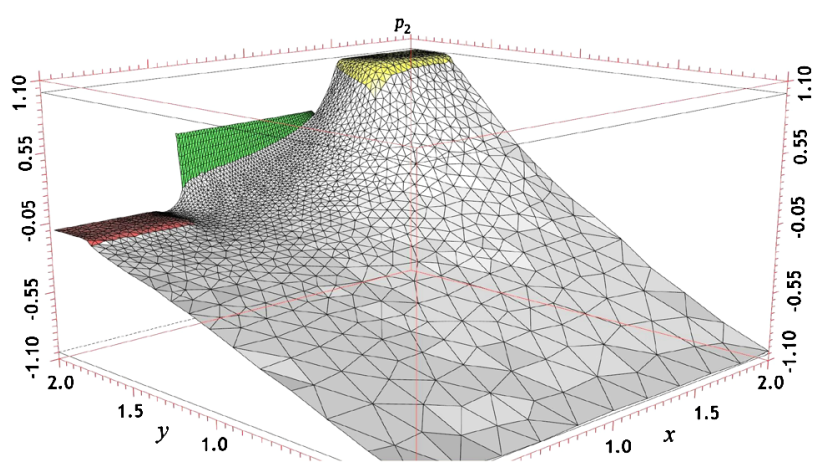

Figure 7 The 3D image of classical SPDMFE solution $p_{2 d}^{n}$ at $t=2$.

because the degrees of freedom of the optimized SPDMFE extrapolation approach (Problem V) are much fewer than those of the classical SPDMFE formulation.

Figure 8 expresses the relative errors between 20 solutions $\left(u_{d}^{n}, \boldsymbol{p}_{d}^{n}\right) \equiv\left(u_{d}^{n}, p_{1 d}^{n}, p_{2 d}^{n}\right)$ of the optimized SPDMFE extrapolation approach with 20 different POD bases and the solutions $\left(u_{h}^{n}, \boldsymbol{p}_{h}^{n}\right) \equiv\left(u_{h}^{n}, p_{1 h}^{n}, p_{2 h}^{n}\right)$ of the classical SPDMFE method at $t=2$, respectively. This implied that when the numbers of POD bases was larger than five, the errors do not exceed $4 \times$ $10^{-4}$. Therefore, the numerical errors above are accordant with theoretical ones. 
Figure 8 When $t=2$, the relative errors between solutions of Problem V with different number of POD bases for a group of 20 snapshots and the classical SPDMFE formulation Problem IV with piecewise first degree polynomial polynomials and piecewise first degree vectors.

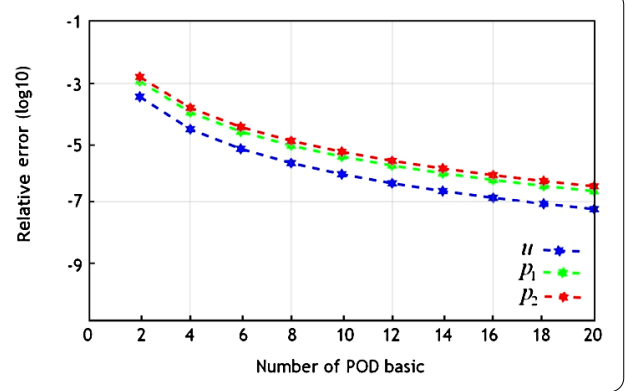

Table 1 RMSEs between the usual POD SPDMFE and optimized SPDMFE extrapolation solutions

\begin{tabular}{lllll}
\hline & $\boldsymbol{N}=\mathbf{5 0}$ & $\boldsymbol{N}=\mathbf{1 0 0}$ & $\boldsymbol{N}=\mathbf{1 5 0}$ & $\boldsymbol{N}=\mathbf{2 0 0}$ \\
\hline$u$ & $1.48 \mathrm{E}-4$ & $2.24 \mathrm{E}-4$ & $3.37 \mathrm{E}-4$ & $4.68 \mathrm{E}-4$ \\
$p_{1}$ & $1.12 \mathrm{E}-4$ & $2.35 \mathrm{E}-4$ & $3.64 \mathrm{E}-4$ & $4.72 \mathrm{E}-4$ \\
$p_{2}$ & $1.38 \mathrm{E}-4$ & $2.23 \mathrm{E}-4$ & $3.36 \mathrm{E}-4$ & $4.82 \mathrm{E}-4$ \\
\hline
\end{tabular}

Table 2 CORCOEs between the usual POD SPDMFE and optimized SPDMFE extrapolation solutions

\begin{tabular}{lllll}
\hline & $\boldsymbol{N}=\mathbf{5 0}$ & $\boldsymbol{N}=\mathbf{1 0 0}$ & $\boldsymbol{N}=\mathbf{1 5 0}$ & $\boldsymbol{N}=\mathbf{2 0 0}$ \\
\hline$u$ & $1.57 \mathrm{E}-4$ & $2.38 \mathrm{E}-6$ & $2.46 \mathrm{E}-8$ & $2.59 \mathrm{E}-9$ \\
$p_{1}$ & $1.46 \mathrm{E}-4$ & $2.35 \mathrm{E}-6$ & $2.65 \mathrm{E}-8$ & $2.71 \mathrm{E}-9$ \\
$p_{2}$ & $1.43 \mathrm{E}-4$ & $2.24 \mathrm{E}-6$ & $2.58 \mathrm{E}-8$ & $2.86 \mathrm{E}-9$ \\
\hline
\end{tabular}

In order to quantify the efficiency of the optimized SPDMFE extrapolation approach, we compare the root mean square errors (RMSE) and the correlation coefficients (CORCOE) between the usual POD SPDMFE solutions with five POD bases (formulated by all 200 SPDMFE solutions on $0 \leq t \leq 2$ ) and the optimized SPDMFE extrapolation solutions with five POD bases (formulated only by the first 20 SPDMFE solutions) at $t=$ $0.5,1.0,1.5$, and 2.0 (i.e., $N=50,100,150$, and 200), respectively. RMSE and CORCOE are, respectively, obtained by the following formulas:

$$
\begin{aligned}
& \operatorname{RMSE}\left(r_{N}\right)=\sqrt{\frac{1}{N} \sum_{n=1}^{N}\left|\tilde{r}_{d}^{n}-r_{d}^{n}\right|^{2}}, \quad r=u, p_{1}, p_{2}, N=50,100,150,200 ; \\
& \operatorname{CORCOE}\left(r_{N}\right)=\frac{\sum_{n=1}^{N}\left(\tilde{r}_{d}^{n}-\overline{\tilde{r}}_{d}^{n}\right)\left(r_{d}^{n}-\bar{r}_{d}^{n}\right)}{\sqrt{\sum_{n=1}^{N}\left(\tilde{r}_{n}^{n}-\overline{\tilde{r}}_{d}^{n}\right)^{2} \sum_{n=1}^{N}\left(r_{d}^{n}-\bar{r}_{d}^{n}\right)^{2}}}, \\
& r=u, p_{1}, p_{2}, N=50,100,150,200,
\end{aligned}
$$

where $\tilde{r}_{d}^{n}\left(r=u, p_{1}, p_{2}\right)$ are the usual POD SPDMFE solutions, $r_{d j}^{n}$ the optimized SPDMFE extrapolation solutions, and $\bar{r}_{d}^{n}$ the mean.

Tables 1 and 2 are, respectively, RMSEs and CORCOEs between the usual POD SPDMFE solutions and the optimized SPDMFE extrapolation solutions at $t=0.5,1.0,1.5$, and 2.0 (i.e., $N=50,100,150$, and 200) with five POD bases. Table 1 shows that the numerically computed RMSEs are consistent with theoretical errors even if they increase with time step numbers. Table 2 also shows that the CORCOEs of the numerical solutions for two cases of the usual POD SPDMFE solutions and the optimized SPDMFE extrapolation solu- 
tions get smaller and smaller with time increasing, which is reasonable since the optimized SPDMFE extrapolation approach only took the first 20 SPDMFE solutions as snapshots. However, the RMSEs are within the tolerance range. Therefore, the optimized SPDMFE extrapolation approach is an improvement over the usual POD SPDMFE model.

By comparing the classical SPDMFE method with the optimized SPDMFE extrapolation approach containing five bases in implementing the numerical simulations when $t=2$, we found that the classical FD scheme at each time level has more than $3 \times 4 \times 10^{4}$ degrees of freedom and requires about 90 minutes computing time on a ThinkPad E530 PC, whereas the RMSEs with five POD bases at each time level only involves $2 \times 5$ degrees of freedom and the corresponding time is about 30 seconds on the same PC, i.e., the computing time of the classical SPDMFE method is about 180 times that of the optimized SPDMFE extrapolation approach with five POD bases. We also showed that the optimized SPDMFE extrapolation approach can greatly reduce the accumulation of the truncated error in the process, diminish the calculation load, save time of calculations, and improve the accuracy of the numerical solutions.

\section{Conclusions and perspective}

In this article, the optimized SPDMFE extrapolation approach based on the POD technique for 2DVWE has been set up, the error estimates between the classical SPDMFE solutions and the optimized SPDMFE extrapolation solutions and the implement procedure for the optimized SPDMFE extrapolation approach have been offered. A numerical example has validated the correctness of the theoretical conclusions, which has expressed that the optimized SPDMFE extrapolation approach is a further development and improvement over the existing methods (see, e.g., [11-16]).

Our future work in this field will aim at developing the optimized SPDMFE extrapolation approach, applying it to several more complicated real-world engineering problems.

Competing interests

The authors declare that they have no competing interests.

Authors' contributions

All authors contributed equally and significantly in writing this article. All authors read and approved the final manuscript.

Author details

'School of Mathematics and Physics, North China Electric Power University, No. 2, Bei Nong Road, Changping District, Beijing, 102206, China. ${ }^{2}$ School of Control and Computer Engineering, North China Electric Power University, No. 2, Bei Nong Road, Changping District, Beijing, 102206, China.

\section{Acknowledgements}

This research was supported by National Science Foundation of China grant (11271127 and 11671106).

Received: 23 October 2016 Accepted: 22 December 2016 Published online: 05 January 2017

References

1. Gurtin, M, Pipkin, A: A general theory of heat conduction with finite wave speeds. Arch. Ration. Mech. Anal. 31, $113-126(1968)$

2. Suveika, K: Mixed problems for an equation describing the propagation of disturbances in viscous media. J. Differ. Equ. 19, 337-347 (1982)

3. Gao, LP, Liang, D, Zhang, B: Error estimates for mixed finite element approximations of the viscoelasticity wave equation. Math. Methods Appl. Sci. 27, 1997-2016 (2004)

4. Li, HR, Luo, ZD, Li, Q: Generalized difference methods and numerical simulation for two-dimensional viscoelastic problems. Math. Numer. Sin. 29(3), 257-262 (2007)

5. Pani, AK, Yuan, JY: Mixed finite element methods for a strongly damped wave equation. Numer. Methods Partial Differ. Equ. 17, 105-119 (2001)

6. Liu, Y, Li, H, Gao, W, He, S, Wang, JF: Splitting positive definite mixed element method for viscoelasticity wave equation. Front. Math. China 7(4), 725-742 (2012) 
7. Li, XC, Sun, P, An, J, Luo, ZD: A new splitting positive definite mixed element formulation for viscoelastic equation. Math. Numer. Sin. 35(1), 45-58 (2013)

8. Holmes, P, Lumley, JL, Berkooz, G: Turbulence, Coherent Structures, Dynamical Systems and Symmetry. Cambridge University Press, Cambridge (1996)

9. Fukunaga, K: Introduction to Statistical Recognition. Academic Press, New York (1990)

10. Jolliffe, IT: Principal Component Analysis. Springer, Berlin (2002)

11. Kunisch, K, Volkwein, S: Galerkin proper orthogonal decomposition methods for parabolic problems. Numer. Math. 90, 117-148 (2001)

12. Luo, ZD, Zhou, YJ, Yang, XZ: A reduced finite element formulation based on proper orthogonal decomposition for Burgers equation. Appl. Numer. Math. 59(8), 1933-1946 (2009)

13. Luo, ZD, Zhu, J, Wang, RW, Navon, IM: Proper orthogonal decomposition approach and error estimation of mixed finite element methods for the tropical Pacific Ocean reduced gravity model. Comput. Methods Appl. Mech. Eng. 196(41-44), 4184-4195 (2007)

14. Luo, ZD, Chen, J, Navon, IM, Yang, XZ: Mixed finite element formulation and error estimates based on proper orthogonal decomposition for the non- stationary Navier-Stokes equations. SIAM J. Numer. Anal. 47(1), 1-19 (2008)

15. Luo, ZD, Li, H, Shang, YQ, Fang, ZC: A LSMFE formulation based on proper orthogonal decomposition for parabolic equations. Finite Elem. Anal. Des. 60, 1-12 (2012)

16. Du, J, Zhu, J, Luo, ZD, Navon, IM: An optimizing finite difference scheme based on proper orthogonal decomposition for CVD equations. Int. J. Numer. Methods Biomed. Eng. 27(1), 78-94 (2011)

17. Luo, ZD, Li, H: A POD reduced-order SPDMFE extrapolating algorithm for hyperbolic equations. Acta Math. Sci. 34B(3), 872-890 (2014)

18. Liu, Q, Teng, F, Luo, ZD: A reduced-order extrapolation algorithm based on CNLSMFE formulation and POD technique for two-dimensional Sobolev equations. Appl. Math. J. Chin. Univ. Ser. A 29(2), 171-182 (2014)

19. Luo, ZD: A POD-based reduced-order stabilized Crank-Nicolson MFE formulation for the no-stationary parabolized Navier-Stokes equations. Math. Model. Anal. 20(3), 346-368 (2015)

20. Adams, RA: Sobolev Spaces. Academic Press, New York (1975)

21. Brezzi, F, Fortin, M: Mixed and Hybrid Finite Element Methods. Springer, New York (1991)

22. Luo, ZD: Mixed Finite Element Methods and Applications. Chinese Science Press, Beijing (2006)

23. Rudin, W: Functional Analysis, 2nd edn. McGraw-Hill, New York (1973)

\section{Submit your manuscript to a SpringerOpen ${ }^{\ominus}$ journal and benefit from:}

- Convenient online submission

Rigorous peer review

- Immediate publication on acceptance

- Open access: articles freely available online

- High visibility within the field

- Retaining the copyright to your article 